\title{
Slow NMDA-Mediated Excitation Accelerates Offset-Response Latencies Generated via a Post- Inhibitory Rebound Mechanism
}

\author{
Ezhilarasan Rajaram,, ${ }^{1,2}$ Carina Kaltenbach, ${ }^{3}$ Matthew J. Fischl,, ${ }^{1, *}$ Leander Mrowka, ${ }^{1}$ Olga Alexandrova, \\ Benedikt Grothe, ${ }^{1}$ Matthias H. Hennig, ${ }^{3}$ and Conny Kopp-Scheinpflug ${ }^{1}$
}

https://doi.org/10.1523/ENEURO.0106-19.2019

${ }^{1}$ Division of Neurobiology, Department Biology II, Ludwig-Maximilians-University Munich, Planegg-Martinsried 82152, Germany, ${ }^{2}$ Graduate School of Systemic Neurosciences, Ludwig-Maximilians-University Munich, Planegg-Martinsried 82152, Germany, and ${ }^{3}$ Institute for Adaptive and Neural Computation, School of Informatics, University of Edinburgh, Edinburgh EH8 9AB, United Kingdom

\begin{abstract}
In neural circuits, action potentials (spikes) are conventionally caused by excitatory inputs whereas inhibitory inputs reduce or modulate neuronal excitability. We previously showed that neurons in the superior paraolivary nucleus (SPN) require solely synaptic inhibition to generate their hallmark offset response, a burst of spikes at the end of a sound stimulus, via a post-inhibitory rebound mechanism. In addition SPN neurons receive excitatory inputs, but their functional significance is not yet known. Here we used mice of both sexes to demonstrate that in SPN neurons, the classical roles for excitation and inhibition are switched, with inhibitory inputs driving spike firing and excitatory inputs modulating this response. Hodgkin-Huxley modeling suggests that a slow, NMDA receptor (NMDAR)-mediated excitation would accelerate the offset response. We find corroborating evidence from in vitro and in vivo recordings that lack of excitation prolonged offset-response latencies and rendered them more variable to changing sound intensity levels. Our results reveal an unsuspected function for slow excitation in improving the timing of post-inhibitory rebound firing even when the firing itself does not depend on excitation. This shows the auditory system employs highly specialized mechanisms to encode timing-sensitive features of sound offsets which are crucial for soundduration encoding and have profound biological importance for encoding the temporal structure of speech.
\end{abstract}

Key words: auditory development; duration encoding; gap-detection; level-tolerance; sound-offset encoding; superior paraolivary nucleus

\section{Significance Statement}

Temporal features like sound-onset and sound-offset responses are crucial for acoustic pattern recognition and vocal communication. In contrast to onset responses, offset responses can be generated solely from inhibition via post-inhibitory rebounds. Here, we demonstrate that excitatory inputs are nevertheless present at offset-encoding neurons of the auditory brainstem where they serve to shorten the response latency of the offset response and stabilize the offset latency against changes in stimulus level.

\section{Introduction}

The relative strength and temporal interaction of excitatory and inhibitory synaptic inputs determine neuronal temporal firing patterns in many parts of the brain including the auditory pathway (Wehr and Zador, 2003; Sun et al., 2010; Denève and Machens, 2016). Acoustic pat-
Received March 20, 2019; accepted May 2, 2019; First published May 31, 2019.

The authors declare no competing financial interests.
Author contributions: E.R., C.K., M.J.F., L.M., O.A., and C.K.-S. performed research; E.R., C.K., M.J.F., O.A., and C.K.-S. analyzed data; E.R., B.G., M.H.H., and C.K.-S. wrote the paper; M.H.H. and C.K.-S. designed research. 
tern recognition and vocal communication require precise processing of the temporal structure of sounds, such as the distinct detection of onsets and offsets, which are encoded by two dissociable channels in the auditory pathway (Anderson and Linden, 2016; Gómez-Álvarez et al., 2018; Kopp-Scheinpflug et al., 2018).

In previous studies of the central auditory system, sound-offset responses were often ignored (KoppScheinpflug et al., 2018). While sound offsets are typically less abrupt (Cavaco and Lewicki, 2007) and more easily obscured by reverberation than onsets, and perceptually less prominent than onsets (Phillips et al., 2002; Deneux et al., 2016; Sohoglu and Chait, 2016), this view is changing with the discovery of neurons dedicated to detecting offsets. Recent studies suggest that neural representations of sound transients, such as offsets, are important for speech perception (Eggermont, 2015), are implicated in auditory dysfunction in brain disorders (Felix et al., 2018) and appear to be involved in short-term memory formation during auditory task performance (Elgueda et al., 2019). A previous study showed that neurons responding to sounds in a sustained fashion do not encode the end of a stimulus as reliably as those which respond specifically to the offset (Kopp-Scheinpflug et al., 2011). Detection of the end of a sound stimulus using the last sound-evoked spike in a neuron with a sustained response is highly variable, mainly due to cellular and synaptic properties changing during stimulus time. At stimulus onset, a neuron is in resting conditions and typically highly excitable, which allows fast and precise spiking. After a period of tonic activity, synaptic depression and activation of voltage-gated conductances reduce excitability, leading to less precise responses (cf. Kopp-Scheinpflug et al., 2011, their Fig. 7). Thus, a careful examination of auditory offset responses will aid in understanding their underlying coding mechanisms, both of which are prerequisites to study their behavioral relevance.

Sound-evoked offset responses have been reported in all processing stages of the ascending auditory pathway from brainstem to cortex (Kopp-Scheinpflug et al., 2018). Offset responses in higher areas such as the auditory cortex seem to be largely inherited from subcortical structures via nonoverlapping sets of excitatory inputs (Scholl et al., 2010). The de-novo generation of sound-offset responses has so far been best described for neurons in the superior olivary complex $(\mathrm{SOC})$ of the mammalian

This work was supported by German Research Council (DFG) Grants KO2207/3-1, SFB870-A10, and GS-82 Graduate School of Systemic Neurosciences GSN ${ }^{\mathrm{LMU}}$

${ }^{*}$ M. J. Fischl's present address: National Institutes of Health, Section on Neuronal Circuitry, Bethesda, MD 20814.

Acknowledgements: We thank Dr. Jennifer Linden and Dr. James Sinclair for helpful discussions and invaluable experimental support.

Correspondence should be addressed to Conny Kopp-Scheinpflug at cks@bio.Imu.de.

https://doi.org/10.1523/ENEURO.0106-19.2019

Copyright (C) 2019 Rajaram et al.

This is an open-access article distributed under the terms of the Creative Commons Attribution 4.0 International license, which permits unrestricted use, distribution and reproduction in any medium provided that the original work is properly attributed. auditory brainstem which exhibit acoustically-evoked offset firing at the end of a sound stimulus (Grothe, 1994; Kuwada and Batra, 1999; Behrend et al., 2002; Dehmel et al., 2002; Kulesza et al., 2003; Kulesza, 2008). These offsets can be generated via a post-inhibitory rebound mechanism that is initiated by strong glycinergic inputs and aided by the activation of hyperpolarization-activated cyclic nucleotide-modulated currents $\left(\mathrm{I}_{H}\right)$ and T-type calcium currents (Felix et al., 2011; Kopp-Scheinpflug et al., 2011). The main source of the glycinergic input triggering these offset responses is the medial nucleus of the trapezoid body (MNTB; Grothe, 1994; Kulesza et al., 2007), which on stimulation evokes IPSCs (Kopp-Scheinpflug et al., 2011) reversing at voltages around $-100 \mathrm{mV}$, well below the neurons' resting membrane potential (Löhrke et al., 2005; Yassin et al., 2014).

It has been shown in in vivo measurements that this strong inhibition is essential for generating short-latency offset responses in a substantial population of SOC neurons (cat: Guinan et al., 1972; bat: Grothe, 1994; rabbit: Kuwada and Batra, 1999; gerbil: Behrend et al., 2002; Dehmel et al., 2002; rat: Kulesza et al., 2003; mouse: Kopp-Scheinpflug et al., 2011; Felix et al., 2013), but the anatomic location and number of SOC neurons with offset responses varies across mammalian species and experimental approaches. In rodents, neurons with offset responses are concentrated in the superior paraolivary nucleus (SPN) where current-clamp recordings in vitro show offset firing in nearly $100 \%$ of neurons (KoppScheinpflug et al., 2011; Felix et al., 2013), while SPN recordings in vivo revealed more differential response types (Behrend et al., 2002; Dehmel et al., 2002; KoppScheinpflug et al., 2011; Felix et al., 2013). These discrepancies in observing different response types between in vivo and in vitro data suggest that so far, in vitro experiments have missed factors that might modulate offset responses and generate more diverse response types.

Excitatory responses of SPN neurons in vivo have been reported either as increased sound-evoked firing rates without obvious inhibition (Behrend et al., 2002; Dehmel et al., 2002) or as responses masked by inhibition, only to be revealed after pharmacological blockade of glycinergic inputs (Kulesza et al., 2007; Jalabi et al., 2013). However, the relevance of excitatory inputs to these predominantly offset-responding neurons for auditory processing is unknown. Octopus cells of the ventral cochlear nucleus have been suggested as one source of SPN excitation based on the contralateral origin of the sound-evoked excitation, its broad frequency tuning (Dehmel et al., 2002) and anatomic tracing experiments (Thompson and Thompson, 1991; Schofield, 1995; Saldaña et al., 2009; Felix et al., 2017). An in vitro study also reports the presence of AMPA receptor (AMPAR)-mediated responses in the mouse SPN (Felix and Magnusson, 2016), but their function and mechanism of action are still unknown.

To gain better insight into the functional relevance of excitatory inputs during sound-offset encoding we performed immunocytochemistry, single-cell recording in vivo, computational modeling, and patch-clamp recordings in vitro. We demonstrate that the time course of slow 
Table 1. Primary and secondary antibodies used for immunocytochemistry

\begin{tabular}{|c|c|c|c|c|c|}
\hline Primary antibody & Antigene & Supplier & Catalog number & Host & Dilution \\
\hline GlyT2 & $\begin{array}{l}\text { Synthetic from the } \mathrm{C} \text { terminus as predicted } \\
\text { from the cloned rat GLYT2 }\end{array}$ & Millipore & $\mathrm{AB} 1773$ & Guinea pig & $1: 1000$ \\
\hline GlyT2 & Recombinant protein (aa1-229 of rat GlyT2) & SySy & 272003 & Rabbit & $1: 1000$ \\
\hline MAP2 & Purified MAP2 isolated from bovine brain & Acris & TA336617 & Chicken & $1: 500$ \\
\hline VGLUT1 & $\begin{array}{l}\text { Purified recombinant protein of rat } \\
\text { VGLUT } 1 \text { (aa456-560) }\end{array}$ & SySy & 135304 & Guinea pig & $1: 2000$ \\
\hline VGLUT3 & $\begin{array}{l}\text { Peptide (C)ELNHEAFVSPRKK, corresponding } \\
\text { to amino acid residues 533-545 } \\
\text { of rat VGLUT3 (accession Q7TSF2); } \\
\text { cytoplasmic, C terminus }\end{array}$ & $\begin{array}{c}\text { Alomone } \\
\text { Labs }\end{array}$ & AGC-037 & Rabbit & $1: 300$ \\
\hline Secondary antibody & Host species & Supplier & Catalog number & Conjugated & Dilution \\
\hline Rabbit & Donkey & Dianova & $711-165-152$ & Су3 & $1: 300$ \\
\hline
\end{tabular}

NMDA receptor (NMDAR)-mediated excitation extends into the temporal window of post-inhibitory rebound firing. Simultaneous activation of excitation and inhibition accelerates post-inhibitory rebound responses and makes them more tolerant against changes in sound intensity in vivo, which is a prerequisite for soundduration tuning and level-independent gap detection (Forrest and Green, 1987).

\section{Materials and Methods}

All experimental procedures were reviewed and approved by the Bavarian district government (TVV AZ: 55.2-1-54-2532-38-13) and were done according to the European Communities Council Directive (2010/63/EU). C57BI6J mice were housed in a vivarium with a normal light dark cycle (12/12 h light/dark) and food and water ad libitum. Mice of both sexes were used for the physiologic and anatomic experiments.

\section{Immunohistochemistry}

Mice (P21-P35) were anesthetized with an overdose of pentobarbital and perfusion-fixed with $4 \%$ paraformaldehyde (PFA) intracardially. Following overnight postfixation in 4\% PFA, coronal brainstem sections including the cochlear nucleus and the SOC of $50-\mu \mathrm{m}$ thickness were taken using a vibrating microtome (Leica Biosystems, VT1200S). After $3 \times 10-$ min washes in PBS, sections were transferred to a blocking solution containing $1 \%$ bovine serum albumin, $0.5 \%$ Triton $\mathrm{X}-100$, and $0.1 \%$ saponin in PBS. For NMDAR staining, proteinase $\mathrm{K}$ treatment (1: 1000 in PBS) for 20 min at $37^{\circ} \mathrm{C}$ was included before transferring the sections into blocking solution. Tissue was incubated for $48 \mathrm{~h}$ at $4^{\circ} \mathrm{C}$ with primary antibodies (Table 1) diluted in blocking solution. Biocytin was labeled with streptavidin conjugated to Cy3 (1:500 in blocking solution). Tissue was then washed $3 \times 10$ min in PBS at room temperature, before incubation for $24 \mathrm{~h}$ at $4^{\circ} \mathrm{C}$ with secondary antibodies diluted in blocking solution. Then sections were rinsed $3 \times 10 \mathrm{~min}$ in PBS, and coverslipped with Vectashield mounting medium.

\section{Confocal microscopy}

Confocal optical sections were acquired with a confocal laser-scanning microscope equipped with HCX PL APO CS 20X/NA0.7 and HCX PL APO Lambda Blue 63×/ NA1.4 immersion oil objectives (Leica). Fluorochromes were visualized with excitation wavelengths of $405 \mathrm{~nm}$ (emission filter $410-430 \mathrm{~nm}$ ) for amino-methylcoumarin (AMCA), $488 \mathrm{~nm}$ (emission filter 510-540 nm) for Alexa Fluor 488, $561 \mathrm{~nm}$ (emission filter 565-585 nm) for Cy3, and $594 \mathrm{~nm}$ (emission filter 605-625 nm) for Alexa Fluor 594. For each optical section, the images were collected sequentially for the different fluorochromes. Stacks of 8-bit grayscale images were obtained with axial distances of $290 \mathrm{~nm}$ between optical sections and pixel sizes of $120-1520 \mathrm{~nm}$ depending on the selected zoom factor and objective. To improve the signal-to-noise ratio, images were averaged from three successive scans. RGB stacks, montages of RGB optical sections and maximumintensity projections were assembled using the ImageJ 1.37k plugins and Adobe Photoshop 8.0.1 software.

\section{In vivo physiology}

Young adult (6-16 weeks) mice of either sex $(n=9)$ were anesthetized with a subcutaneous injection of 0.01 $\mathrm{ml} / \mathrm{g}$ MMF $(0.5 \mathrm{mg} / \mathrm{kg}$ body weight medetomidine, 5.0 $\mathrm{mg} / \mathrm{kg}$ body weight midazolam, and $0.05 \mathrm{mg} / \mathrm{kg}$ body weight fentanyl) and were placed on a temperaturecontrolled heating pad (WPI: ATC 1000) in a soundproof chamber (Industrial Acoustics). Depth of anesthesia was measured using the toe pinch reflex and animals responding were given supplemental MMF at 1/3 the initial dose. The mice were then stabilized in a custom stereotaxic device. An incision was made at the top of the skull, and a head post was fixed to the skull using dental cement. A craniotomy was performed above the cerebellum to ac- 
cess the auditory brainstem. A ground electrode was placed in the muscle at the base of the neck. Glass microelectrodes were pulled from glass capillaries so that the resistance was 5-20 M $\Omega$ when filled with $3 \mathrm{M} \mathrm{KCl}$ solution or $2 \mathrm{M}$ potassium acetate with $2.5 \%$ biocytin. Signals were amplified (AM Systems, Neuroprobe 1600), filtered (300-3000 Hz; Tucker-Davis-Technologies PC1), and recorded ( $\sim 50 \mathrm{kHz}$ sampling rate) with an RZ6 processor (Tucker-Davis Technologies). Python-based SPIKE software (Brandon Warren, V.M. Bloedel Hearing Research Center, University of Washington) was used to calibrate the multi-field magnetic speakers, generate stimuli and record action potentials. Stimuli consisted of pure tones (50- to 100 -ms duration, 5-ms rise/fall time) at varying intensity (0to $90-\mathrm{dB}$ SPL) and were presented through hollow ear bars connected to the speakers with Tygon tubing. PSTHs were assessed at characteristic frequency (CF) and 80-dB SPL. Spike sorting and data analysis were performed offline using custom MATLAB programs. At the end of the experiment, biocytin (2.5\%) was deposited at the final penetration using the current injection mode of the amplifier $(+0.5 \mu \mathrm{A}, 1-2$ $\mathrm{min}$ ). Thirty minutes were allowed for cellular uptake before the animal was perfused, and the tissue was processed for biocytin fluorescence as described above. Recording sites were determined using the biocytin deposition as a reference for stereotaxic reconstruction.

\section{In vitro electrophysiology}

Mice of either sex P15-P22 were briefly anaesthetized with isoflurane and rapidly decapitated. Coronal brainstem sections (150-200 $\mu \mathrm{m}$ thick) containing the SOC were cut in an ice-cold high-sucrose, low-sodium artificial CSF (ACSF). Brainstem slices were maintained after slicing in normal ACSF at $37^{\circ} \mathrm{C}$ for $30-45 \mathrm{~min}$, after which they were stored in a slice-maintenance chamber at room temperature $\left(\sim 22^{\circ} \mathrm{C}\right)$. Composition of the normal ACSF: $125 \mathrm{mM} \mathrm{NaCl}, 2.5 \mathrm{mM} \mathrm{KCl}, 26 \mathrm{mM} \mathrm{NaHCO}{ }_{3}, 10 \mathrm{mM}$ glucose, $1.25 \mathrm{mM} \mathrm{NaH} \mathrm{PO}_{4}, 2 \mathrm{mM}$ sodium pyruvate, 3 $\mathrm{mM}$ myo-inositol, $2 \mathrm{mM} \mathrm{CaCl}_{2}, 1 \mathrm{mM} \mathrm{MgCl}_{2}$, and $0.5 \mathrm{mM}$ ascorbic acid, $\mathrm{pH} 7.4$, bubbled with $95 \% \mathrm{O}_{2}, 5 \% \mathrm{CO}_{2}$. For the low-sodium ACSF $\mathrm{CaCl}_{2}$ and $\mathrm{MgCl}_{2}$ concentrations were 0.1 and $4 \mathrm{mM}$, respectively, and $\mathrm{NaCl}$ was replaced by $200 \mathrm{mM}$ sucrose. Experiments were conducted at 36 $\pm 1^{\circ} \mathrm{C}$, maintained by an inline feedback temperature controller and heated stage (Warner Instruments) with the recording chamber being continuously perfused with ACSF at a rate of $1-2 \mathrm{ml} \mathrm{min}^{-1}$. Whole-cell patch-clamp recordings were made from visually identified SPN neurons using an EPC10/2HEKA amplifier (HEKA Electronik), sampling at $50 \mathrm{kHz}$ and filtering between 2.9 and $10 \mathrm{kHz}$. Patch pipettes were pulled from borosilicate glass capillaries (Warner Instruments) using a DMZ Universal electrode puller (Zeitz-Instuments Vertriebs $\mathrm{GmbH}$ ), filled with a patch solution containing: $126 \mathrm{mM} \mathrm{K}$-gluconate, $4 \mathrm{mM}$ $\mathrm{KCl}, 40 \mathrm{mM}$ HEPES, EGTA $5 \mathrm{mM}$ HEPES, $1 \mathrm{mM} \mathrm{MgCl}_{2}, 5$ $\mathrm{mM} \mathrm{Na}{ }_{2}$ phosphocreatine, $0.2 \%$ biocytin, $292 \mathrm{mOsm}, \mathrm{pH}$ was adjusted to 7.2 with $\mathrm{KOH}$. For recordings of EPSCs the internal solution contained: $135 \mathrm{mM}$ Cs-gluconate, $10 \mathrm{mM}$ HEPES, $1 \mathrm{mM}$ EGTA, $3.3 \mathrm{mM} \mathrm{MgCl}_{2}, 3 \mathrm{mM}$ $\mathrm{Na}_{2}$ phosphocreatine, 2 mM NaATP, 20 mM TEA-Cl, 0.2\% biocytin, $300 \mathrm{mOsm}$. pH was adjusted to 7.2 with $\mathrm{CsOH}$. Data were corrected for liquid junction potentials of -13.8 and $-13.7 \mathrm{mV}$ for the potassium-based and the cesiumbased internal solutions, respectively. Electrode resistance was between 2.4 and $6 \mathrm{M} \Omega$. Synaptic responses were evoked by afferent fiber stimulation with either concentric or bipolar (FHC) electrodes. Voltage pulses were generated by the HEKA amplifier and postamplified by an isolated pulse stimulator (AM Systems). Synaptic conductances were calculated from the synaptic currents: $G=P S C /\left(V_{m}-E_{P S C}\right)$, with $P S C$ being the postsynaptic current, $V_{m}$ being the holding potential (-60 mV for inhibition; $40 \mathrm{mV}$ for excitation), $\mathrm{E}_{\mathrm{PSC}}$ being the reversal potential of the postsynaptic current $\left(E_{\mathrm{E}^{-}}\right.$ PSC: $0 \mathrm{mV}$; $\mathrm{E}_{\mathrm{IPSC}}$ : $-100 \mathrm{mV}$ ).

\section{Computational model}

Two simple, single-compartment models of a SPN neuron were simulated using NEURON (version 7.5; Hines and Carnevale, 2001). In both models, the basic set-up of the neuron, including the membrane properties and the ionic channels, is identical to the model developed by Kopp-Scheinpflug et al. (2011), available on ModelDB (https://senselab.med.yale.edu/modeldb/ShowModel. asp? model=139657), accession number 139657. In the two current models, the excitatory noise was removed, and four excitatory synapses were added in addition to the inhibitory synapses already present. The synapses are modeled using the NEURON built-in function Exp2Syn. In both models, the excitatory and inhibitory stimuli consist of 10 such spikes with 10 -ms gaps, resulting in a total stimulus duration of $100 \mathrm{~ms}$. The excitatory and inhibitory conductances and the respective time constants used in the model are provided by the patch-clamp experiments performed for this paper. In the first model (including AMPA currents), all four excitatory synapses are simulating AMPA synapses, with a reversal potential $E_{\text {rev_AMPA }}=$ $0 \mathrm{mV}$, a rise time constant of $\tau_{1}=0.1 \mathrm{~ms}$ and a decay time constant of $\tau_{2}=0.9 \mathrm{~ms}$. In the second model (including AMPA and NMDA currents), only two excitatory synapses are simulating AMPA synapses, with the same parameters as in the first model; while the two other excitatory synapses are simulating simplified NMDA synapses, with reversal potential $E_{\text {rev NMDA }}=0 \mathrm{mV}$, a rise time constant of $\tau_{1}=3 \mathrm{~ms}$, and a decay time constant of $\tau_{2}=9 \mathrm{~ms}$. The conductances of the excitatory synapses were varied in both models. Both models included 14 inhibitory synapses with reversal potential $E_{\text {rev_inh }}=-100 \mathrm{mV}$, a rise time constant $\tau_{1}=0.1 \mathrm{~ms}$, a decay time constant $\tau_{2}=$ $2 \mathrm{~ms}$ and a peak conductance of $82 \mathrm{~ns}$ and $41 \mathrm{nS}$. A simplified depression of the input synapses was modeled using the steady-state depression values collected for this paper.

\section{Experimental design and statistical analysis}

In the text data are presented in parenthesis as (median; $25 / 75$ quartiles or as mean \pm SEM; test: $p$ value) unless indicated otherwise. In the figures, data are presented as medians (lines in boxes); $25 / 75$ quartiles (boxes); and 10th/90th percentiles (whiskers) in addition to individual data points. Statistical analyses of the data 
were performed with SigmaStat/SigmaPlot. Normality was tested by the Shapiro-Wilk test. Comparisons between different data sets were made depending on the distribution of the data using parametric tests for normally distributed data (two-tailed Student's $t$ test for comparing two groups and ANOVA for comparing three or more groups). When the normality assumption was violated, nonparametric tests (Mann-Whitney rank-sum test for comparing two groups and Kruskal-Wallis ANOVA on ranks for comparing three or more groups) were used. Paired $t$ tests or Wilcoxon signed rank tests were used when two data sets were recorded from individual neurons under different conditions. Differences were considered statistically significant at $p \leq 0.05$ and presented in the figures as n.s. for nonsignificant differences and as $* p$ $\leq 0.05, * * p \leq 0.01$, and $* * * p \leq 0.001$ for significant differences. Intrinsic properties as well as postsynaptic current amplitudes and kinetics were analyzed using Stimfit software (Guzman et al., 2014). For data acquired with in vivo single-unit recording or patch-clamp recording; $n$ is the number of neurons, with two to three brain slices per animal and at least three animals per group.

\section{Results}

\section{SPN neurons receive glycinergic and glutamatergic synaptic input}

We previously showed that only inhibitory synaptic input is required to generate offset responses in the SPN (Kopp-Scheinpflug et al., 2011). Here, this was corroborated by immunocytochemistry showing strong expression of the neuronal glycine transporter type 2 (GlyT2), which labels glycinergic synaptic terminals around the soma of SPN neurons (Fig. 1, green boutons). However, the additional presence of excitatory synapses was confirmed by labeling for the vesicular glutamate transporter (VGLUT) types 1-3 (Fig. 1A,D,G). While VGLUT1 and VGLUT2 positively labeled presynaptic terminals in SPN (Fig. 1B,C,E,F), the signal strength for VGLUT3 was quite low and somatic rather than in the presynaptic boutons (Fig. $1 H, l)$.

\section{SPN neurons with sound-offset responses can exhibit moderate excitatory responses during contralateral sound stimulation}

To explore the contribution of excitatory inputs to signal processing in neurons with sound-offset responses, spikes were recorded from single SPN neurons in anesthetized mice in vivo, during (peristimulus) and after (poststimulus) the presentation of sound (Fig. $2 A$ ). The sample of 20 SPN neurons had CFs ranging from 9.25 to $50.4 \mathrm{kHz}$ (Fig. 2B); 85\% (17/20) of these neurons showed a burst of increased firing at the end of contralateral sound stimulation (offset responses) and little or no firing during sound presentation (Fig. 2C,D), consistent with a prevalent inhibitory input and the dominance of offset responses reported in previous studies (Dehmel et al., 2002; Kulesza et al., 2003; Felix et al., 2013). Only 3/20 SPN neurons did not exhibit offset firing, but responded with an onset, primary-like or sustained firing pattern during sound stim- ulation (Fig. 2E). These neurons that fired spikes only during but not after the stimulus were not included in further analyses. Of SPN neurons with offset responses, $53 \%(9 / 17)$ additionally showed excitatory responses during sound stimulation that exceeded $5 \%$ of the respective neurons' overall firing rate (Fig. $2 F$ ). These neurons will be further referred to as ON-OFF type neurons (Fig. $2 F, G$, gray) and will be contrasted against neurons that exhibit offset responses without peristimulus excitation (OFFonly type; Fig. $2 F$, blue). Average temporal response patterns show ON-OFF type neurons with either an onset or a primary-like temporal response pattern during sound followed by a poststimulus offset response (Fig. 2G, gray histogram). Onset or primary-like temporal response patterns are associated with octopus cells in the ventral cochlear nucleus (Rhode et al., 1983) which are one source of excitatory input to the SPN (Thompson and Thompson, 1991; Schofield, 1995; Saldaña et al., 2009; Felix et al., 2017). The average response of the OFF-only type neurons is characterized by only little spontaneous firing during sound followed by a slightly delayed poststimulus offset response (Fig. 2G, blue histogram). The ratio of peristimulus to poststimulus rate is significantly larger in the ON-OFF type neurons (ON-OFF type: 0.22; $0.12 / 1.39 ; n=9$; OFF-only type: $0 ; 0 / 0.09 ; n=8$; MannWhitney rank-sum test: $p=0.002$; Fig. $2 H$ ).

\section{SPN neurons that exhibit additional excitation have accelerated and level-independent offset-response latencies}

The average temporal response pattern in Figure 2G suggests that offset-response latencies are shorter in ON-OFF type neurons compared to OFF-only type neurons. To investigate potential differences in latency in more detail, offset responses were recorded at CF for increasing sound intensities (Fig. $3 A, B$ ). Indeed, offsetresponse latencies were significantly faster for ON-OFF type neurons (5.55 ms, 3.80/6.97 ms, $n=8$ ) than for OFF-only type neurons $(8.84 \mathrm{~ms}, 6.38 / 16.75 \mathrm{~ms}, n=9$; Mann-Whitney rank-sum test: $p=0.018$; Fig. $3 C$ ). However, no difference was observed for the variability of the offset-response latency (jitter) between ON-OFF and OFF-only type neurons (jitter ${ }_{\text {ON-OFF: }} 1.23 \mathrm{~ms} ; 0.62 / 2.54$

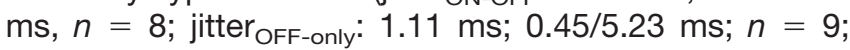
Mann-Whitney rank-sum test: $p=0.665$; Fig. $3 D$ ), suggesting that the intrinsic properties of the cells that regulate precise spike firing are not the reason for the difference in latencies.

A common feature in stimulus encoding across different sensory modalities is that response latencies decrease with increasing stimulus intensity (auditory: Kitzes et al., 1978; Klug et al., 2000; somatosensory: Mountcastle et al., 1957; visual: Morgan and Thompson, 1975). In contrast, offset-response latencies of both ON-OFF type (Fig. $3 A$ ) and OFF-only type (Fig. 3B) SPN neurons did not show a strong dependency on stimulus intensity but remained rather constant over a large sound intensity range or even showed a trend of increasing latencies with increasing intensities (single cell examples shown in Fig. $3 A, B)$. On average, there were no significant changes in 

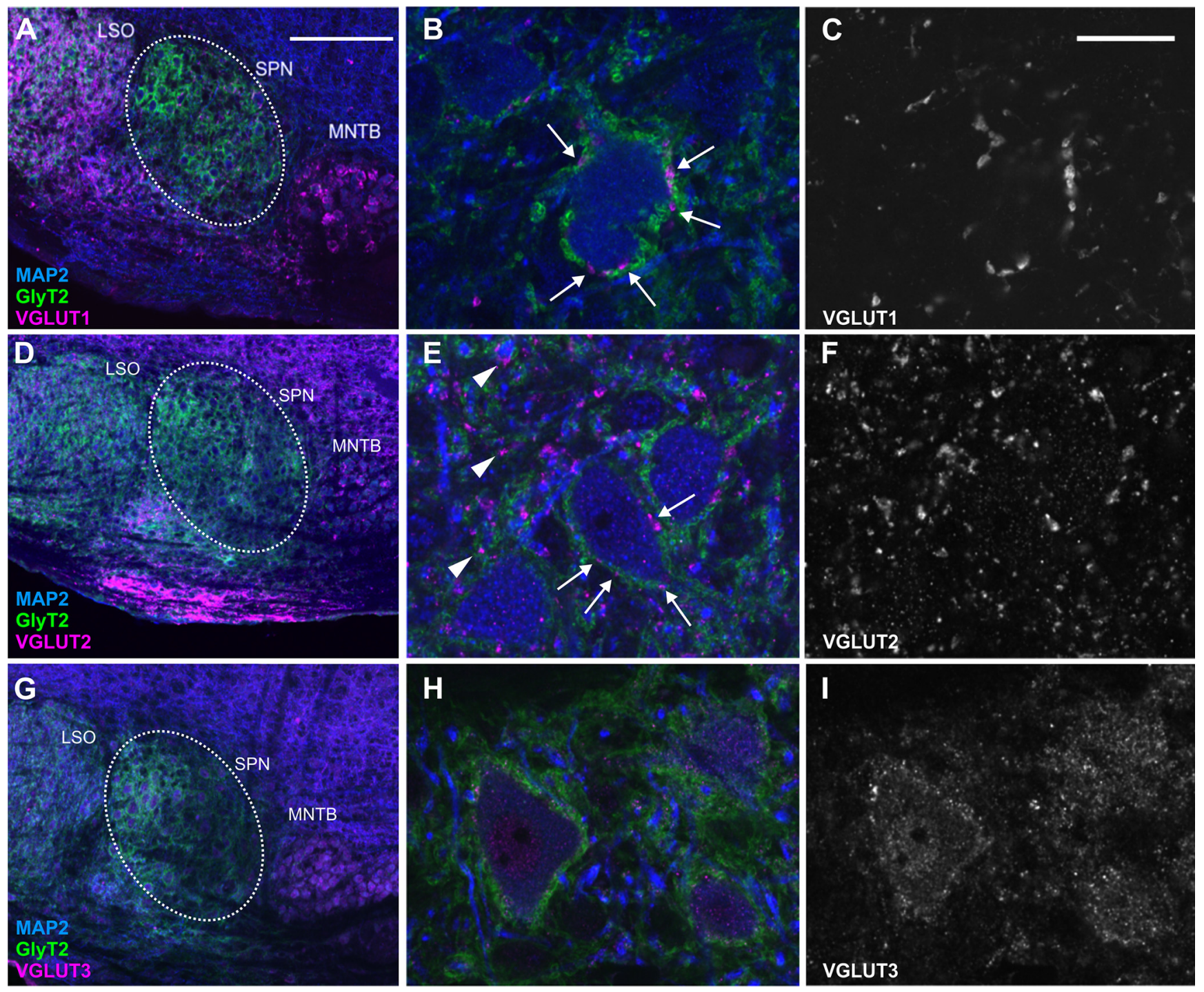

Figure 1. Histochemical profile of excitatory and inhibitory inputs to the SPN. A, B, D, E, G, H, Glycinergic input forms the most prominent input to SPN (white dotted circle in $\boldsymbol{A}, \boldsymbol{D}, \boldsymbol{G}$ ) and is depicted by neuronal GlyT2 (green) labeling. Immunolabeling for microtubule associated protein 2 (MAP2, blue) is used as a neuronal marker in all images. Glutamatergic inputs are shown by labeling for the VGLUTs (magenta): VGLUT1 (A-C), VGLUT2 (D-F), VGLUT3 (G-I). $\boldsymbol{A}-\boldsymbol{F}$, While both VGLUT1 (B, white arrows) and VGLUT2 (E, white arrows) positive synaptic boutons are present at the soma, VGLUT2 boutons are also seen in the neuropil (E, white arrowheads). G-I, VGLUT3 shows only weak somatic but no presynaptic bouton labeling. Scale bars $=200 \mu \mathrm{m}$ (left images) and $20 \mu \mathrm{m}$ (middle and right images).

latency per decibel sound intensity for the ON-OFF type neurons $(-9.83 \mu \mathrm{s} / \mathrm{dB} ;-19.46 / 5.11 \mu \mathrm{s} / \mathrm{dB} ; n=6$; Fig. $3 E)$, while changes in latencies per decibel for the OFF-only type neurons were significantly larger $(-47.79 \mu \mathrm{s} / \mathrm{dB}$; $-67.37 /-13.87 \mu \mathrm{s} / \mathrm{dB} ; n=6$, Kruskal-Wallis ANOVA on ranks followed by Dunn's multiple comparisons versus a zero change control: $p=0.032$ ).

If the neurons firing either ON-OFF or OFF-only responses represent two distinct populations, they may inhabit different locations in the SPN. This was tested by testing whether ON-OFF or OFF-only responding neurons have similar or different CFs. The CFs of the offset response for ON-OFF type neurons (15.1 kHz; 12.0/21.8 $\mathrm{kHz} ; n=8)$ were compared with those for OFF-only type neurons (18.5 kHz; $12.3 / 22.3 \mathrm{kHz} ; n=9$ ) but no significant difference was found (Mann-Whitney rank-sum test: $p=$ 1.000; Fig. 3F). Tonotopically organized glycinergic projections from MNTB into SPN suggest that neurons tuned to high frequencies are located more medially and neurons tuned to low frequencies are located more laterally (Banks and Smith, 1992). As a result, neurons with different CFs should inhabit separate anatomic locations within the tonotopic axis of the SPN, which was not observed in the present sample of ON-OFF and OFF-only type neurons and suggests, that neurons having ON-OFF or OFFonly responses are part of a continuum with the only difference being differently balanced excitation and inhibition. Response thresholds of offset responses were also not significantly different between ON-OFF type (32-dB SPL; 16.3/48.8-dB SPL; $n=8$ ) and OFF-only type neu- 
A

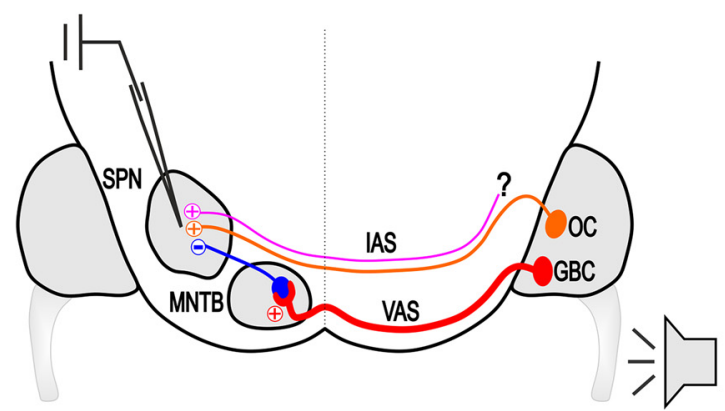

B

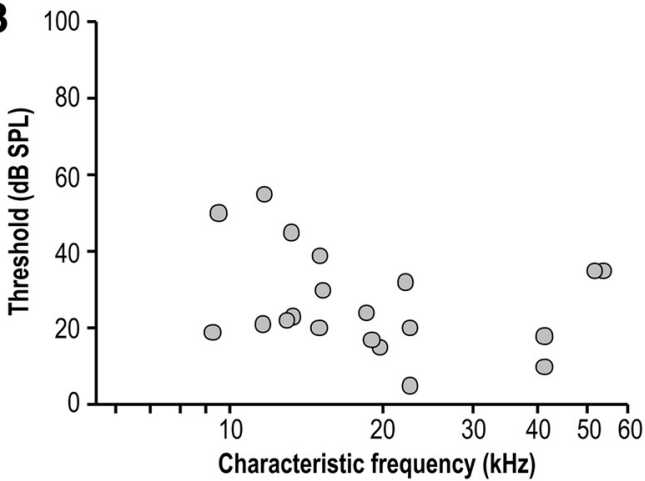

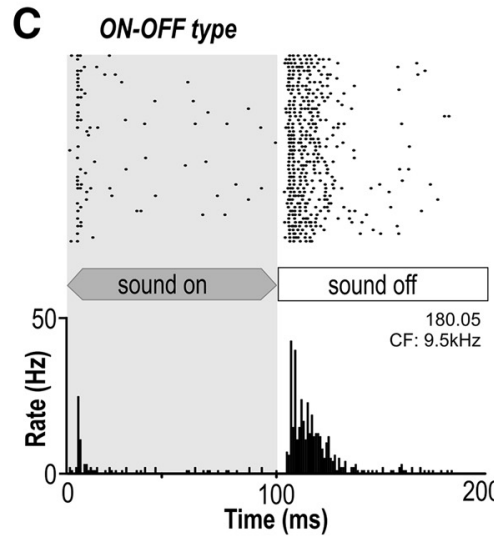

D OFF-only type

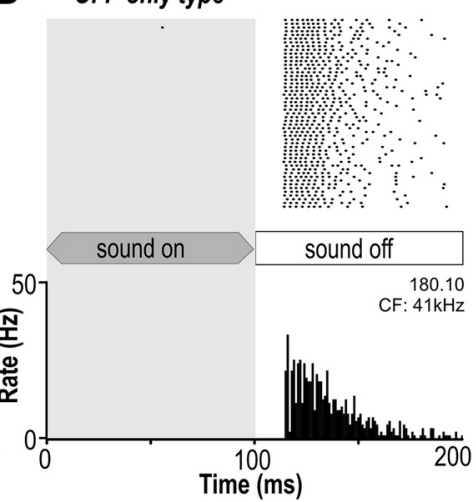

E
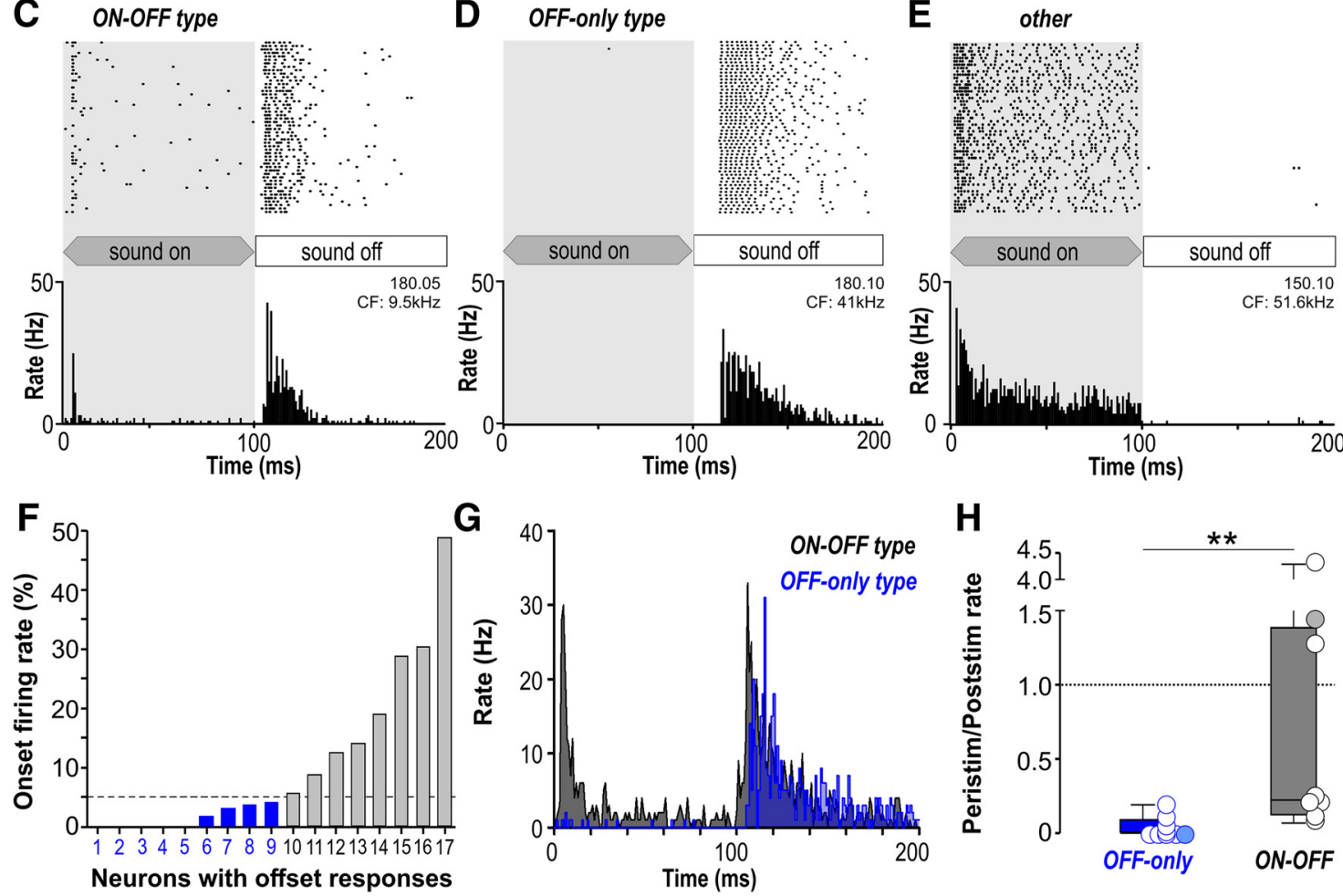

G

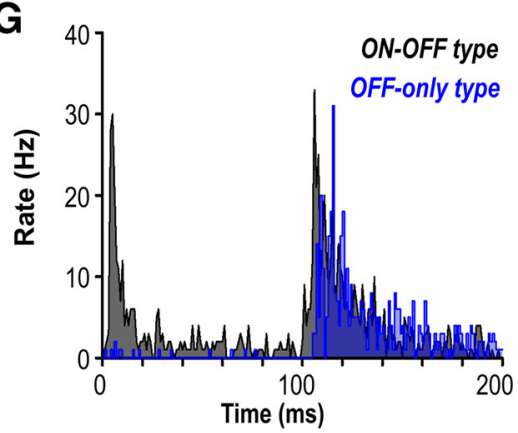

H

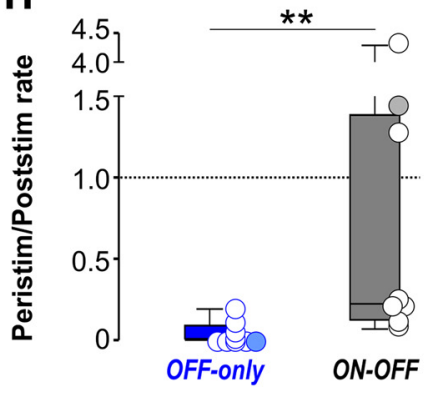

Figure 2. SPN neurons with post-inhibitory rebound responses at sound offset can also show excitatory responses during sound. $\boldsymbol{A}$, Schematic of a sound-offset encoding circuit in the mammalian brainstem. Globular bushy cell (GBC) axons project via the ventral acoustic stria (VAS) to the contralateral MNTB to form one-to-one connections via the giant calyx of Held synapses. MNTB neurons then project to neurons of the SPN. SPN cells also receive excitatory input from octopus cells (OCs) and possibly other, yet unknown sources (?) in the contralateral ventral cochlear nucleus via the IAS. B, Plot of CFs and auditory thresholds of all SPN neurons recorded in this sample. C-E, Raster plots and PSTHs (at CF, 80-dB SPL, 50 trials) shown for three individual SPN neurons with different response patterns. $\boldsymbol{C}$, ON-OFF type, showing a peristimulus response related to sound onset followed by a response related to sound offset. $\boldsymbol{D}$, OFF-only type, showing responses related to sound offset, but no peristimulus firing. $\boldsymbol{E}$, Response type showing only peristimulus firing but no poststimulus response at sound offset. $\boldsymbol{F}$, Distribution of neurons with offset responses depending on how much of their overall firing activity during the combined $200-\mathrm{ms}$ peristimulus and poststimulus period was present within the first 20 $\mathrm{ms}$ of the response. The dashed line represents the $5 \%$ criterion we used to classify the neurons into either OFF-only type neurons (blue: 1-9) or ON-OFF type neurons (gray: 10-17). G. Average temporal response patterns for OFF-only type neurons (blue) and ON-OFF type neurons (gray). $\boldsymbol{H}$, Peristimulus-to-poststimulus ratio for OFF-only type neurons (blue) and ON-OFF type neurons (gray). Firing rates were averaged over the whole peristimulus time window and divided by the average of the whole poststimulus time window. Equal firing rates in both time windows result in a ratio of zero (dotted line). Filled circles represent the ratios for the example cells shown in $\boldsymbol{C}, \boldsymbol{D}$. Note that OFF-only neurons with ratios larger than zero exhibit spontaneous APs which also appear during the peristimulus time window but not concentrated within the first $20 \mathrm{~ms}$ to form an onset response. $* * p \leq 0.01$.

rons (21-dB SPL; 18.5/28-dB SPL; $n=9$; Mann-Whitney rank-sum test: $p=0.386$; Fig. $3 G$ ).

The occurrence of excitatory responses in some but not all neurons of a nucleus with generally dominant inhibition could also be attributed to a reduced inhibitory constraint in ON-OFF type neurons, resulting in higher spontaneous firing rates compared to OFF-only type neurons. Inhibition in SPN neurons is provided by 


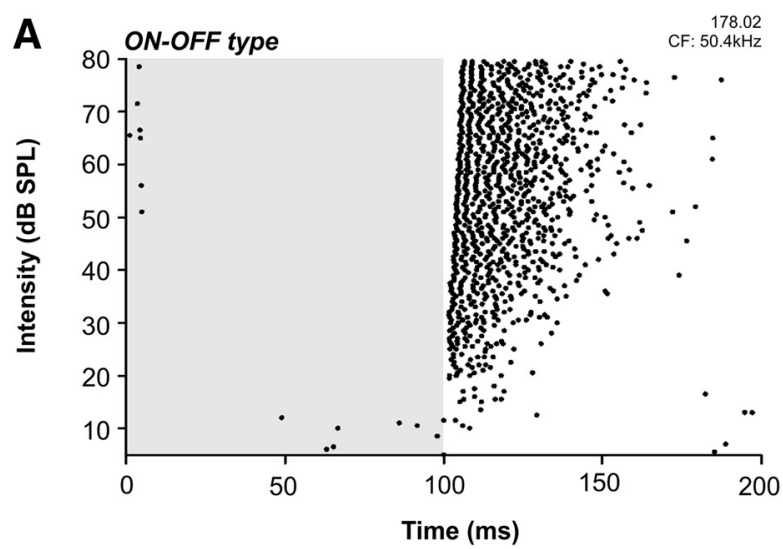

B
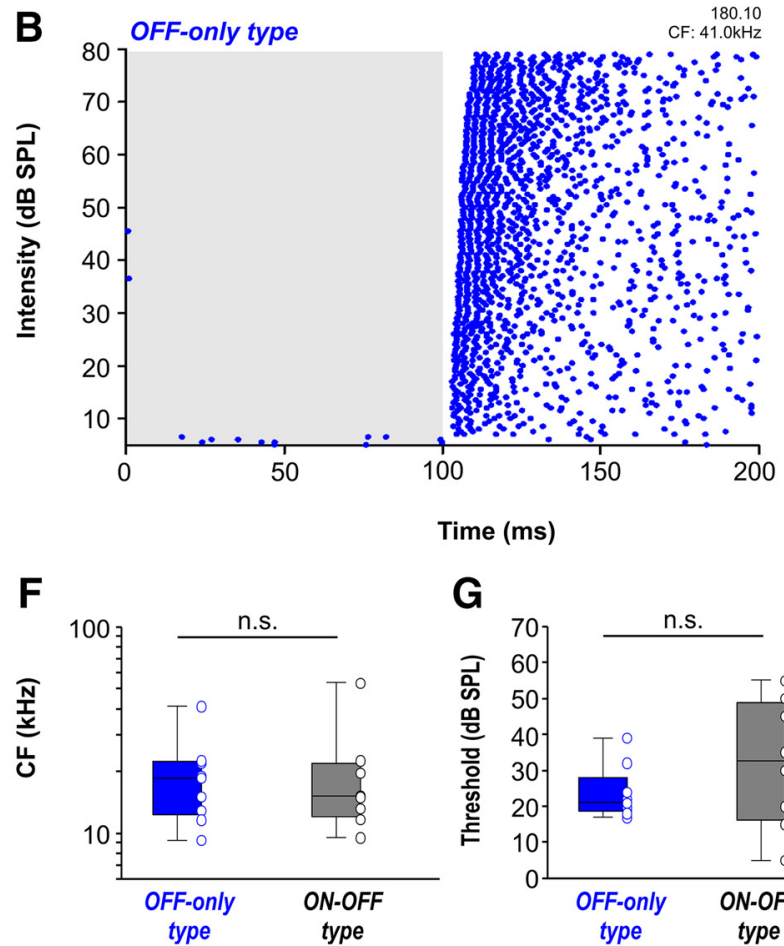

G

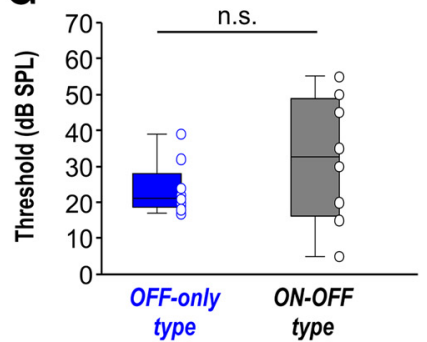

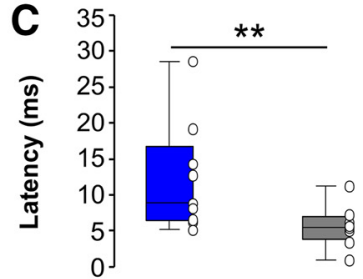
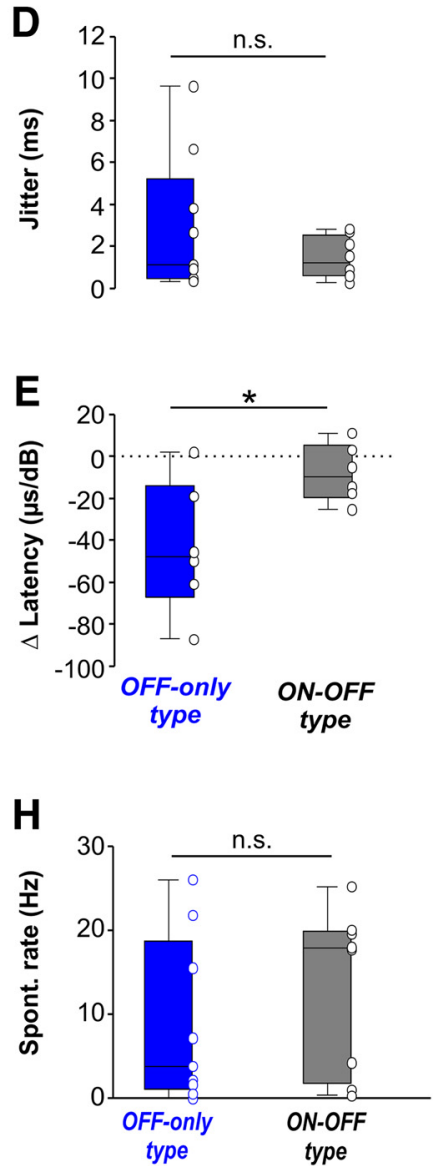

Figure 3. ON-OFF type SPN neurons have shorter offset-response latencies and less level dependence than OFF-only type neurons. $\boldsymbol{A}, \boldsymbol{B}$, Raster plot at CF and changing intensity levels for representative (A) ON-OFF type and (B) OFF-only type neurons. Each dot represents an action potential. Gray-shaded area indicates sound duration. $\boldsymbol{C}, \boldsymbol{D}$, Distribution of $(\boldsymbol{C})$ offset latencies and $(\boldsymbol{D})$ jitter for OFF-only type (blue) and ON-OFF type (gray) neurons at CF/80-dB SPL. Statistical assessment in $\boldsymbol{C}, \boldsymbol{D}$ was unaltered if the two extreme values were removed. $\boldsymbol{E}$, Average change of offset-response latency per dB intensity change for OFF-only type (blue) and ON-OFF type (gray) SPN neurons. OFF-only type neurons (blue) showed a significantly larger variability of offset-response latency with changes in intensity. Latency change/dB was not significantly different from zero (dotted line) for ON-OFF type neurons (=level invariant). $\boldsymbol{F}-\boldsymbol{H}$, Between SPN neurons of either OFF-only type or ON-OFF type, physiologic parameters like $(\boldsymbol{F}) \mathrm{CFs},(\boldsymbol{G})$ thresholds, and $(\boldsymbol{H})$ spontaneous firing rates are not significantly different. $* p \leq 0.05, * * p \leq 0.01$, n.s. $=$ non-significant.

strong glycinergic input from MNTB neurons. Despite high firing rates during sound stimulation, MNTB neurons are spontaneously active with an average rate of 20-30 Hz (Kopp-Scheinpflug et al., 2008) which tonically suppresses SPN activity. Spontaneous firing rates in SPN were not significantly different between ON-OFF type neurons $(13.25 \pm 3.5 \mathrm{~Hz})$ compared to OFF-only type neurons $(8.73 \pm 3.7 \mathrm{~Hz}$; two-tailed $t$ test: $p=$ $0.359 ; t=0.946$; df $=15$; Fig. $3 H$ ), implying a similar strength of inhibitory innervation across neurons. Across the population of cells tested, ON-OFF type and
OFF-only type neurons do not exhibit differences in location or general physiologic properties suggesting them to belong to one cell population with differing strength of excitatory input.

\section{Offset-response thresholds are more sensitive than peristimulus-response thresholds}

Comparing the peristimulus and poststimulus excitatory responses within each ON-OFF type SPN neuron revealed that their spectral tuning largely overlapped (Fig. $4 A, B)$, which is in contrast to results from auditory cortex 

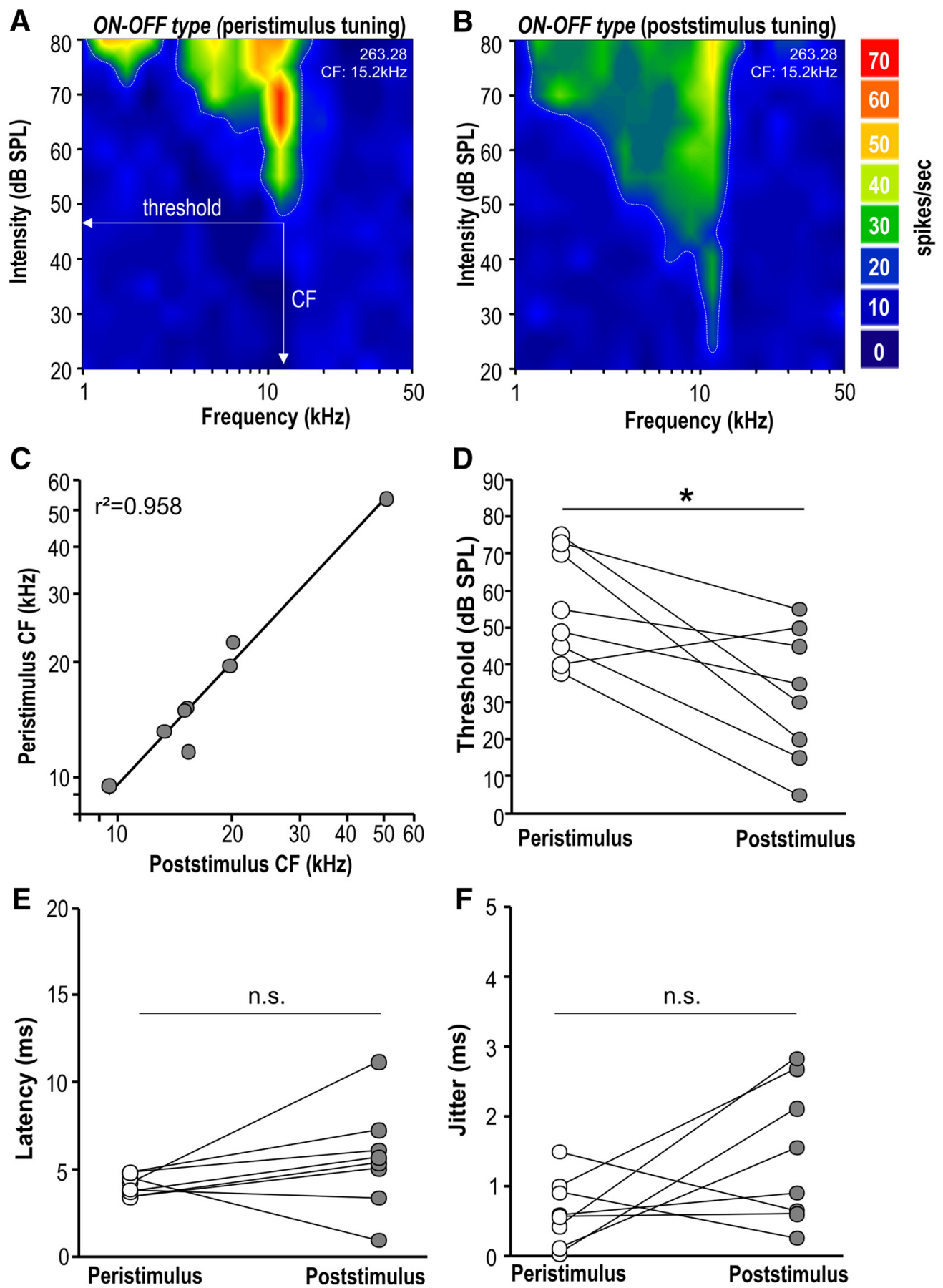

Figure 4. Within ON-OFF type SPN neurons, peristimulus responses have similar tuning, but elevated threshold compared to their poststimulus offset-responses. $\boldsymbol{A}, \boldsymbol{B}$, Frequency-intensity response maps for an individual ON-OFF type SPN neuron plotted for the peristimulus response $(\boldsymbol{A})$ and for the poststimulus response after sound cessation $(\boldsymbol{B})$. $\boldsymbol{C}$, Linear correlation between peristimulus and poststimulus CFs for individual ON-OFF type SPN neurons. $\boldsymbol{D}$, Thresholds for the responses during sound (peristimulus, white circles) are higher than thresholds for responses after sound termination (poststimulus, gray circles). $\boldsymbol{E}$, $\boldsymbol{F}$, Latencies and jitter were not significantly different between peristimulus and poststimulus response of individual ON-OFF type SPN neurons. $* p \leq 0.05$, n.s. $=$ non-significant.

(Scholl et al., 2010; Sollini et al., 2018) and will be discussed later. There was no significant difference between the peristimulus and poststimulus CFs for ON-OFF type neurons ( $\mathrm{CF}_{\text {peristim: }}$ : $19.8 \pm 4.5 \mathrm{kHz} ; n=8$; $\mathrm{CF}_{\text {poststim }}: 20.0$ $\pm 4.9 \mathrm{kHz} ; n=8$; two-tailed paired $t$ test: $p=0.74 ; t=$ -0.345 ; df $=7$; Fig. 4C). Thresholds were significantly lower for poststimulus offset responses (32 \pm 6 -dB SPL) compared to excitatory peristimulus responses (56 \pm 5-dB SPL; $n=8$; two-tailed paired $t$ test: $p=0.011 ; t=$ 3.407; df = 7; Fig. 4D). For each ON-OFF type neuron first-spike latencies for the excitatory peristimulus response $(4.12 \pm 0.2 \mathrm{~ms})$ were barely faster than those of 
A

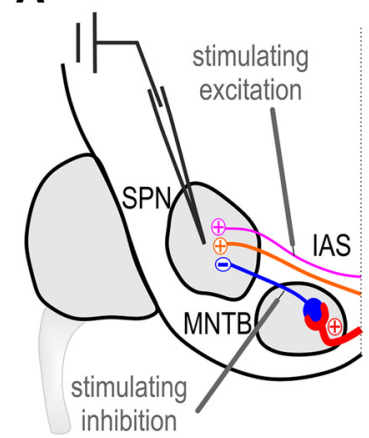

D

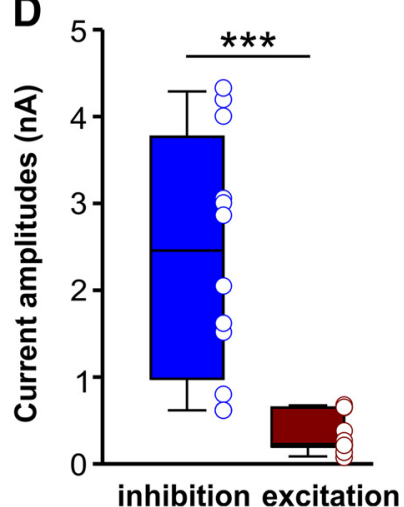

B

SR95531, strych SR95531, strych, DNQX,D-AP5

$-60 \mathrm{mV}$

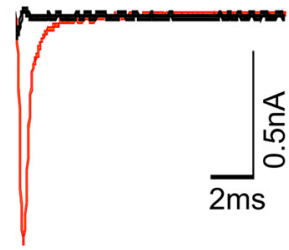

E

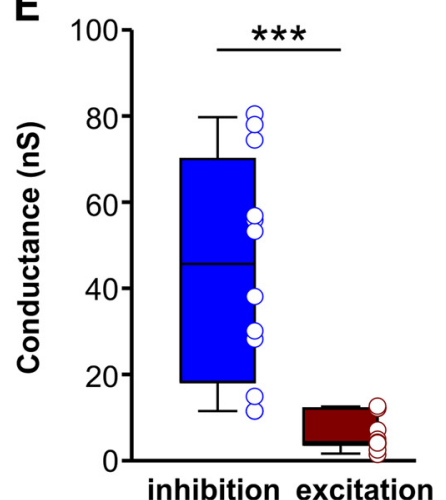

C

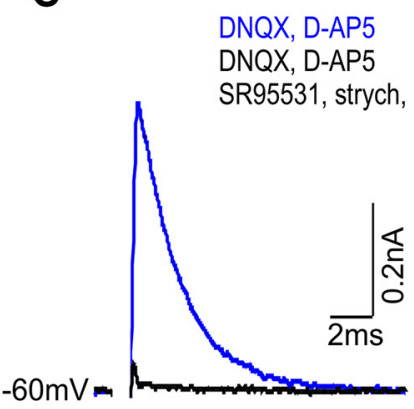

F

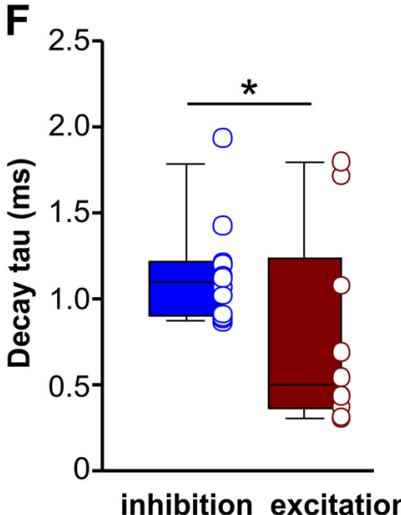

Figure 5. Comparison of synaptic strength between EPSCs and IPSCs in SPN neurons. $\boldsymbol{A}$, Schematic of the sound-offset encoding circuit depicting the positions of the stimulating electrodes for eliciting either excitation or inhibition. $\boldsymbol{B}, \boldsymbol{C}$, Voltage-clamp traces of pharmacologically isolated EPSCs $(\boldsymbol{B})$ evoked by stimulating IAS and IPSCs $(\boldsymbol{C})$ evoked by stimulating MNTB (average of 10 traces). Black traces indicate the blockade of $(\boldsymbol{B})$ EPSCs or (C) IPSCs. $\boldsymbol{D}$, Average IPSC (blue) and EPSC (red) amplitudes measured in response to maximum stimulation. E, EPSCs and IPSCs expressed as conductance reveals that mean IPSG values are more than five times larger than EPSGs. F, Average decay time constants $(\tau)$ of EPSCs and IPSCs. Tau IPSC is significantly slower than Tau $\mathrm{EPSC}_{\mathrm{EPC}} * * p \leq 0.001$.

the offset response following sound cessation $(5.64 \pm 1.0$ ms; two-tailed paired $t$ test: $p=0.188 ; t=1.458 ; \mathrm{df}=7$; Fig. 4E), indicating a high speed in generating offset responses despite the additional synaptic delay arising during the sign conversion in the MNTB. Jitter as a measure of the temporal precision of the first spike in the response was also not significantly different between the peristimulus $(0.64 \pm 0.17 \mathrm{~ms})$ and the poststimulus $(1.45$ $\pm 0.35 \mathrm{~ms}$ ) response (two-tailed paired $t$ test: $p=0.111$; $t=1.822 ; \mathrm{df}=7$; Fig. $4 F$ ). The mean latency of $\sim 4 \mathrm{~ms}$ for the peristimulus response is similar to that of other SOC neurons in mouse (Kopp-Scheinpflug et al., 2008). Although the average temporal response pattern of the ON-OFF type neurons shows a primary-like pattern (Fig. 2G), 5/8 ON-OFF type neurons show onset firing patterns. To investigate whether primary-like or onset-responses can influence the offset response, in vivo recordings of spikes do not provide sufficient information about possible subthreshold activity that lasts throughout the stimulation, which will more appropriately be measured by whole-cell patch-clamp recordings in vitro.

\section{Strength of inhibition outweighs excitation in SPN neurons}

Whole-cell patch-clamp recordings in vitro were made of SPN neurons. Neurons had resting membrane potentials of $-61.74 \pm 0.79 \mathrm{mV}$, an average input resistance of
$74.39 \pm 10.94 \mathrm{M} \Omega$ and an average membrane capacitance of $65.63 \pm 7.78 \mathrm{pF}(n=23)$. Glutamatergic EPSCs were regularly activated in neurons throughout the SPN. For electrical stimulation, a concentric stimulating electrode was placed on the intermediate acoustic stria (IAS) medial to the SPN and just dorsal to the MNTB (Fig. 5A). EPSCs were pharmacologically isolated by adding the $\mathrm{GABA}_{\mathrm{A}}$ receptor blocker SR95531 $(20 \mu \mathrm{M})$ and the glycine receptor blocker strychnine $(1 \mu \mathrm{M})$ to the bath solution (Fig. 5B). Glycinergic IPSCs were evoked by direct electrical stimulation of the ipsilateral MNTB using a concentric stimulating electrode and were pharmacologically isolated by adding the AMPAR blocker 6,7-dinitroquinoxaline-2,3-dione (DNQX; $10 \mu \mathrm{M}$ ) and the NMDAR blocker D-2-amino-5-phosphonopentanoic acid (D-AP5; $50 \mu \mathrm{M}$ ) to the bath solution (Fig. 5C). At physiologic holding voltages near the neurons resting membrane potential (-60 mV), IPSC amplitudes were significantly larger than EPSCs (IPSC: 2.46; 0.98/3.77; $n=12$; EPSC: 0.23; $0.20 / 0.65 ; n=11$; Mann-Whitney rank-sum test: $p \leq$ 0.001; Fig. 5D). The considerable difference between the strength of excitation and that of inhibition was even more obvious when both were expressed as conductances (IPSG: 45.65; 18.22/70.02; $n=12$; EPSG: 4.26; 3.72/ 12.08; $n=11$; Mann-Whitney rank-sum test: $p \leq 0.001$; Fig. $5 E$ ). The decay time constants of IPSCs were significantly slower than that of EPSCs (Tau IPSC: 1.09; 0.90/ 

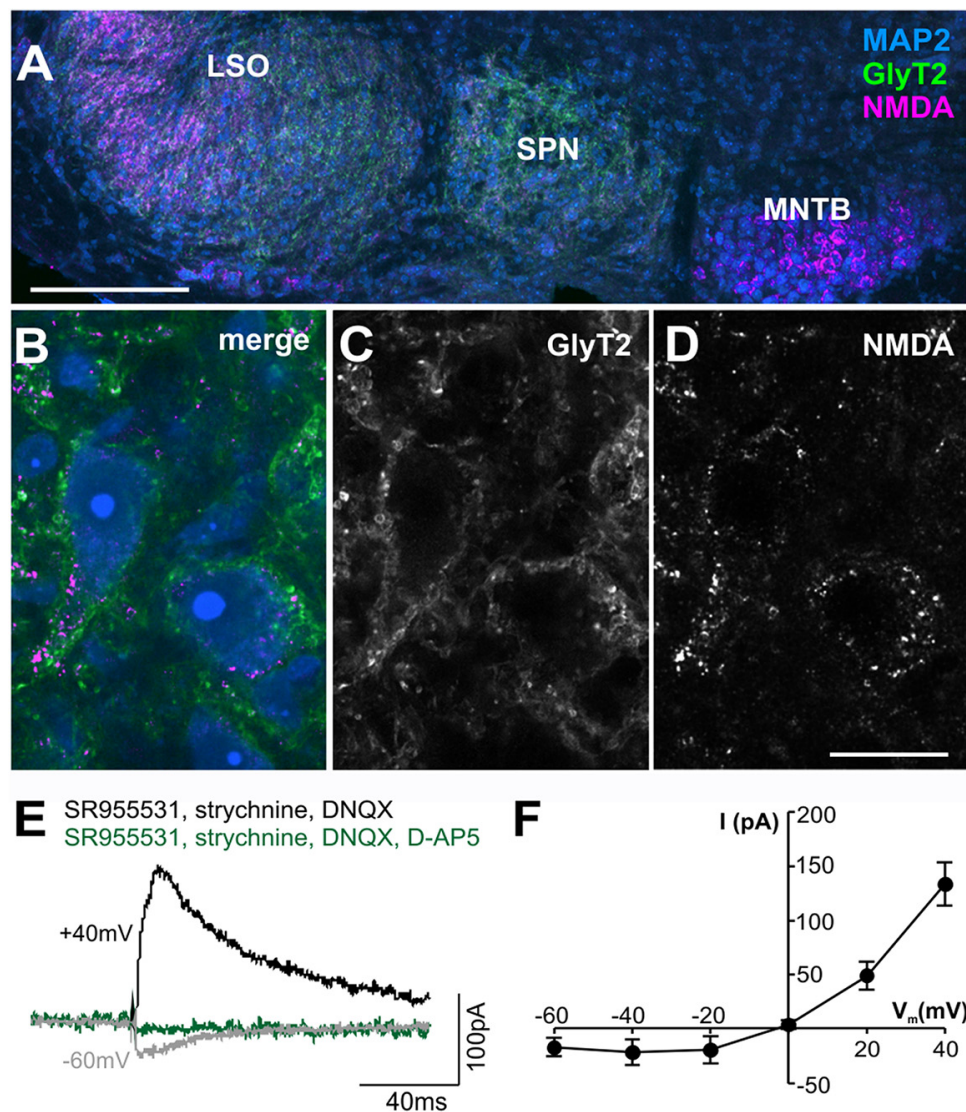

$\mathbf{F}$
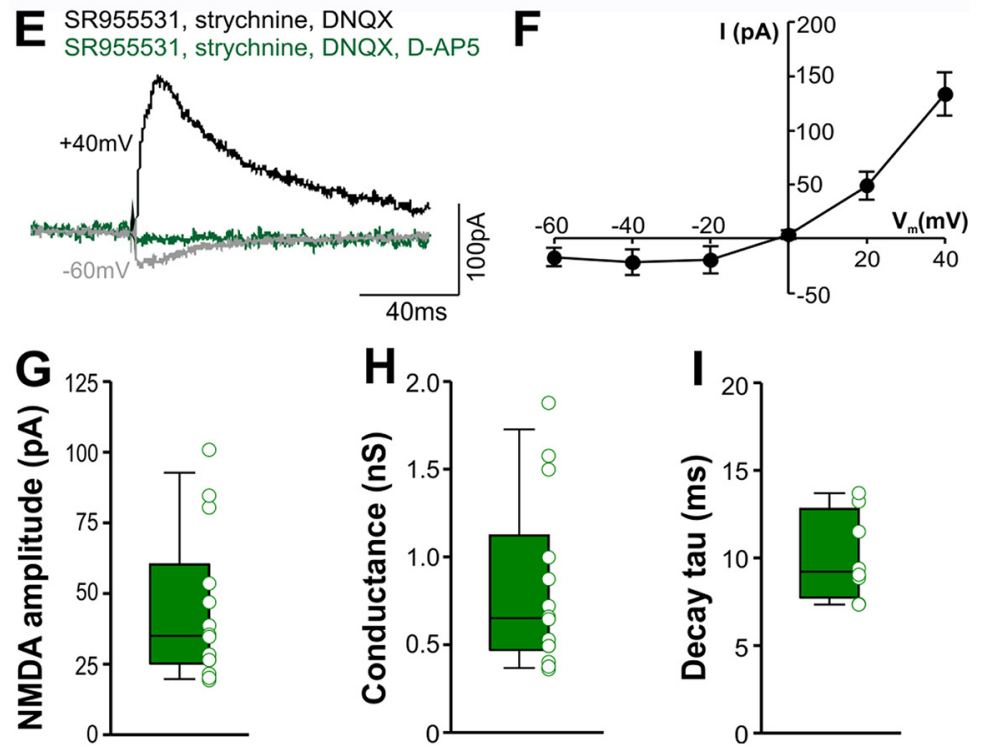

Figure 6. SPN neurons express NMDARs which mediate moderate EPSCs at depolarized voltages. $\boldsymbol{A}$, Low-power image of SOC showing NMDA immunoreactivity (magenta), which was strong in the MNTB and the lateral superior olive (LSO), and to a lesser degree present in the SPN. GlyT2 labeling (green) identifies the outline of the SPN. MAP2 (blue) was used as a neuronal marker. B-D, Higher magnification images show that NMDARs are present in SPN neurons. $\boldsymbol{E}$, Voltage-clamp traces of pharmacologically isolated NMDA currents at $-60 \mathrm{mV}$ (gray) and at $+40 \mathrm{mV}$ (black). Currents were blocked by D-AP5 (green). $\boldsymbol{F}$, Average NMDA currents show the typical nonlinearity due to the $\mathrm{Mg}^{2+}$ block at hyperpolarized membrane voltages. $\mathbf{G}$, Amplitude of NMDA currents at $+40 \mathrm{mV}$. $\boldsymbol{H}$, NMDA conductance. $\boldsymbol{I}$, Decay time constants $(\tau)$ of NMDA currents at $+40 \mathrm{mV}$. Scale bars $=200 \mu \mathrm{m}(\boldsymbol{A})$ and $20 \mu \mathrm{m}(\boldsymbol{B}-\boldsymbol{D})$.

$1.21 ; n=12$; Tau $_{\text {EPSC }}: 0.49 ; 0.36 / 1.24 ; n=11$; MannWhitney rank-sum test: $p=0.038$; Fig. $5 F$ ).

\section{NMDAR-mediated currents are present in SPN neurons}

Glutamate released from excitatory synaptic inputs typically activates AMPAR and/or NMDAR with fast and slow kinetics, respectively. Current-clamp recordings of SPN neurons near the neuronal resting potential suggested that excitatory responses are primarily mediated by AMPARs (Felix and Magnusson, 2016). Here, we used immunocytochemistry to probe for the presence of
NMDARs and performed voltage-clamp recordings to assess the strength of NMDAR-mediated currents at different membrane potentials. NMDAR expression is evident in mature SPN neurons, although weaker compared to neurons in the MNTB or LSO (Fig. 6A-D). Electrophysiological measurement of NMDA currents in whole-cell voltage-clamp mode revealed the characteristic voltage dependence causing larger currents once the membrane voltage reaches depolarized values (Fig. $6 E, F$ ) and showed that they were sensitive to the NMDAR antagonist D-AP5 (Fig. 6E). NMDA currents in SPN neurons (35.0pA; 25.2/60.3pA; $n=14$; Fig. $6 G$ ) were smaller than in MNTB 
(Steinert et al., 2010), but similar to LSO (Alamilla and Gillespie, 2011; Pilati et al., 2016) and MSO (Smith et al., 2000; Couchman et al., 2012). NMDA conductances calculated from these currents ranged from 0.36 to $1.88 \mathrm{nS}$ $(0.65 ; 0.47 / 1.12 n S ; n=14)$. The small, yet prevalent NMDA currents resemble the general decline of NMDA currents in the auditory brainstem following hearing onset. However, as Steinert and colleagues have shown for neurons in the neighboring MNTB, after the initial reduction in amplitudes, NMDA currents reach a steady state with no signs of further decline after about two weeks of age (Steinert et al., 2010), corroborating the presence of NMDA current in the SPN for ages of $15 \mathrm{~d}$ and older. Decay time constants of the NMDAR-mediated EPSCs ranged from 7.34 to $13.69 \mathrm{~ms}(9.21 \mathrm{~ms} ; 7.74 / 12.79 \mathrm{~ms} ; n$ $=8$; Fig. $5 H$ ). In later experiments AMPAR and NMDAR responses will be activated and blocked in unison by a cocktail of DNQX/D-AP5 and together will be contrasted against glycinergic inhibition to reveal the contribution of excitation to ON-OFF type SPN neurons.

\section{Differential short-term plasticity between excitatory and inhibitory SPN synapses}

To test for a balance between excitatory and inhibitory inputs in an adapted, more physiologic state, pharmacologically or electrically isolated inhibitory, excitatory and NMDAR-mediated synaptic currents were evoked in SPN neurons by applying 50-pulse fiber stimulation trains at $100 \mathrm{~Hz}$ (Fig. 7A). Since NMDAR-mediated currents can only be activated at depolarizing potentials positive to the EPSC reversal potential, they are shown as outward currents in the green trace in Figure 7A. For better visualization NMDA currents are flipped in the superimposed enlargement of all three current types for the first $100 \mathrm{~ms}$ of the response (Fig. 7B). Both fast inhibitory and excitatory currents showed pronounced short-term depression, calculated from a train of IPSCs and EPSCs, respectively, and normalized to the first peak (Fig. $7 C, D$ ). In contrast NMDARmediated currents showed very little depression (Fig. 7C,D). The time constant of depression was fastest for EPSCs (1.92 $\mathrm{ms} ; 1.41 / 2.39 \mathrm{~ms} ; n=9)$, a little slower in IPSCs (2.88 ms; 2.53/3.23 ms; $n=17$ ) and very slow in NMDAR-mediated currents (10.31 ms; 4.01/16.45 ms; $n=5$; Kruskal-Wallis ANOVA on ranks followed by Dunn's multiple comparisons; $p \leq 0.001$; Fig. 7E). The average level of steady state depression was similar between EPSCs and IPCSs, but significantly lower in in NMDAR-mediated currents (inhibition: 66.70\%; 62.57/72.05\%; $n=17$; excitation: $74.03 \%$; 71.54/ $81.42 \% ; n=9$; NMDA: $42.36 \% ; 25.87 / 55.40 \% ; n=5$; Kruskal-Wallis ANOVA on ranks followed by Dunn's multiple comparisons; $p \leq 0.001$; Fig. 7F).

Across the SPN, IPSCs were always larger than EPSCs. The position of each recorded neuron within the SPN was logged by taking a picture of the electrode position in $4 \times$ magnification. The SPN was then divided into 4 quadrants, which were correlated to the size of the currents. No correlation was found, suggesting a homogeneous distribution of excitatory inputs across the SPN with no distinct, spatially segregated subpopulations. The moderate, excitatory inputs to SPN neurons become visible as pat- terns of increased firing activity during sound presentation in about half of the offset-responding SPN neurons. The fact that these neurons have peristimulus as well as poststimulus responses raises new questions: do glutamatergic inputs, that drive peristimulus excitatory responses, interact with peristimulus inhibitory inputs that drive the poststimulus offset response, and if so, what is their impact on the offset response? To specifically address the role of NMDAR-mediated currents, compared to AMPAR-mediated currents, we fed the data acquired by voltage-clamp recording into a computational model to simulate both types of excitatory synapses and the inhibitory inputs to an SPN neuron.

\section{Computational modeling suggests that moderate and slow excitation affects offset-response latency}

A basic Hodgkin-Huxley model of SPN neuron firing was developed to test the effect of excitatory inputs of variable strength that are present in addition to inhibitory inputs. All synaptic conductances used for the following simulations were taken from the results presented in Figures $5-7$. Conductance evoked by a single pulse, the synaptic depression during trains of synaptic stimulation and the duration of the stimulus train were taken into consideration while determining the range of conductances to be used in the model. Inhibitory conductances, recorded in vitro after a single pulse ranged from 11.5 to $80.5 \mathrm{nS}$ with a median of $45 \mathrm{nS}$. We estimated the synaptic depression during a $100-\mathrm{Hz}$ stimulation for $100 \mathrm{~ms}$ to be around $50 \%$, resulting in a conductance of $41 \mathrm{nS}$ $(50 \%$ of $80.5 \mathrm{nS})$. For longer stimulation of $500 \mathrm{~ms}$, a steady state depression estimated at $64 \%$, results in minimal possible conductance values of $3.9 \mathrm{nS}(64 \%$ of $11.5 \mathrm{nS}$ ). This is approximately the value where the inhibition becomes strong enough to generate a rebound spike in the model depicted in Figure 8E. Excitatory conductances were subjected to a similar approach, with minimum experimentally acquired excitatory conductances of $0.4 \mathrm{nS}$ (minimal measured value with maximum depression) to $12.6 \mathrm{nS}$ (maximal measured value with no depression). For NMDA currents, the range between the minimal measured value with maximum depression and maximum measured value with no depression was 0.3$1.9 \mathrm{nS}$. However, since NMDA currents depress only little and even initially facilitate (Fig. $7 C, D$ ), an average of $11 \%$ facilitation was added to the maximum measured value, providing an NMDA conductance of $2.1 \mathrm{nS}$. The values from in vitro experiments might be underestimating the synaptic conductances, as axons will be cut during the slicing procedure. To account for this caveat, we take advantage of the model to simulate a broader range of conductances. These physiologically feasible core conductances for inhibition, excitation and NMDA are covered in the matrices in Figure $8 E, F$. When using an inhibition-only model (Kopp-Scheinpflug et al., 2011) with adapting inputs, SPN neurons reliably fired a burst of offset responses following a 100-ms train of IPSCs presented at $100 \mathrm{~Hz}$ (Fig. 8A). The original model was amended in the full model by adding adapting AMPARmediated and NMDAR-mediated currents (Fig. 8B). This 
A
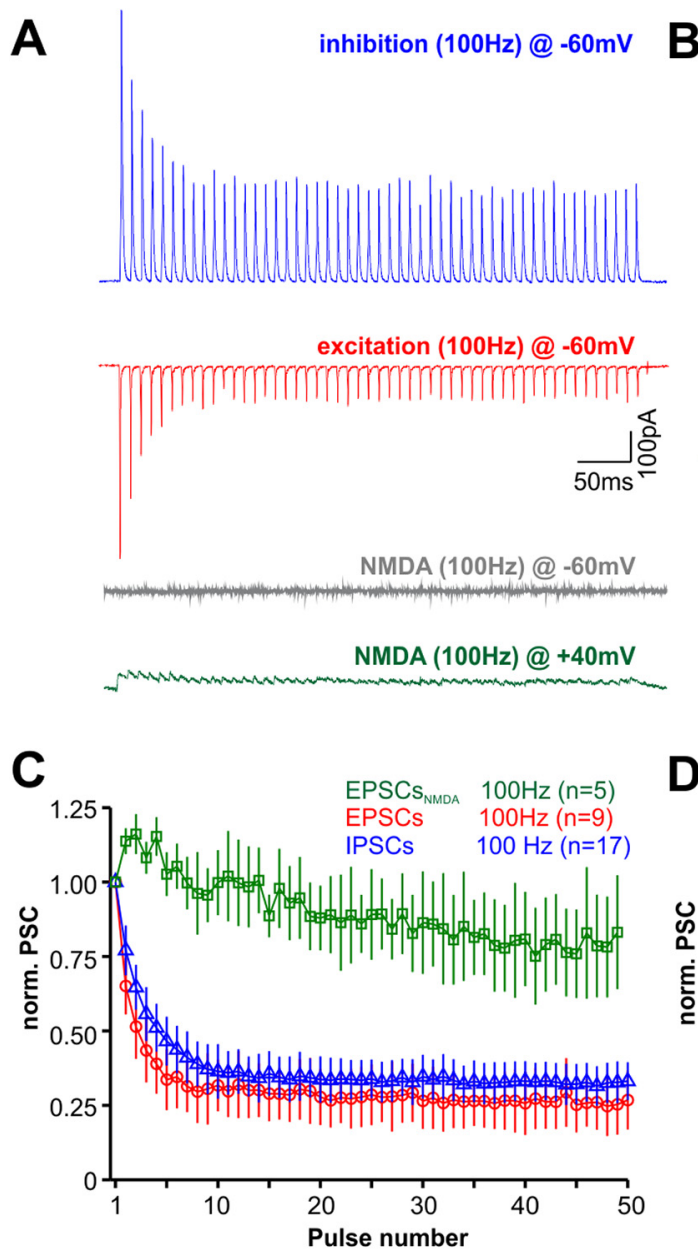

\section{E}

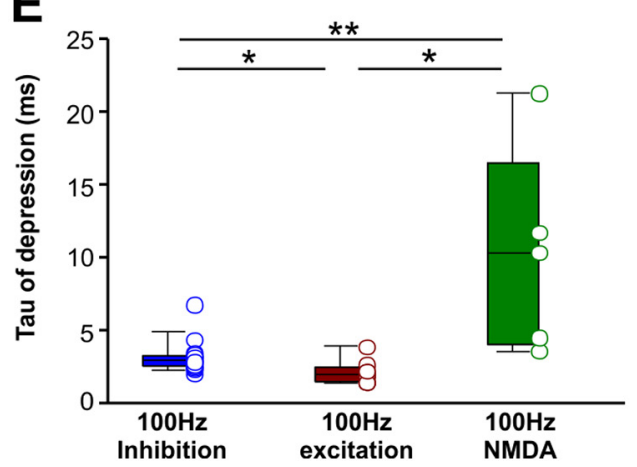

B
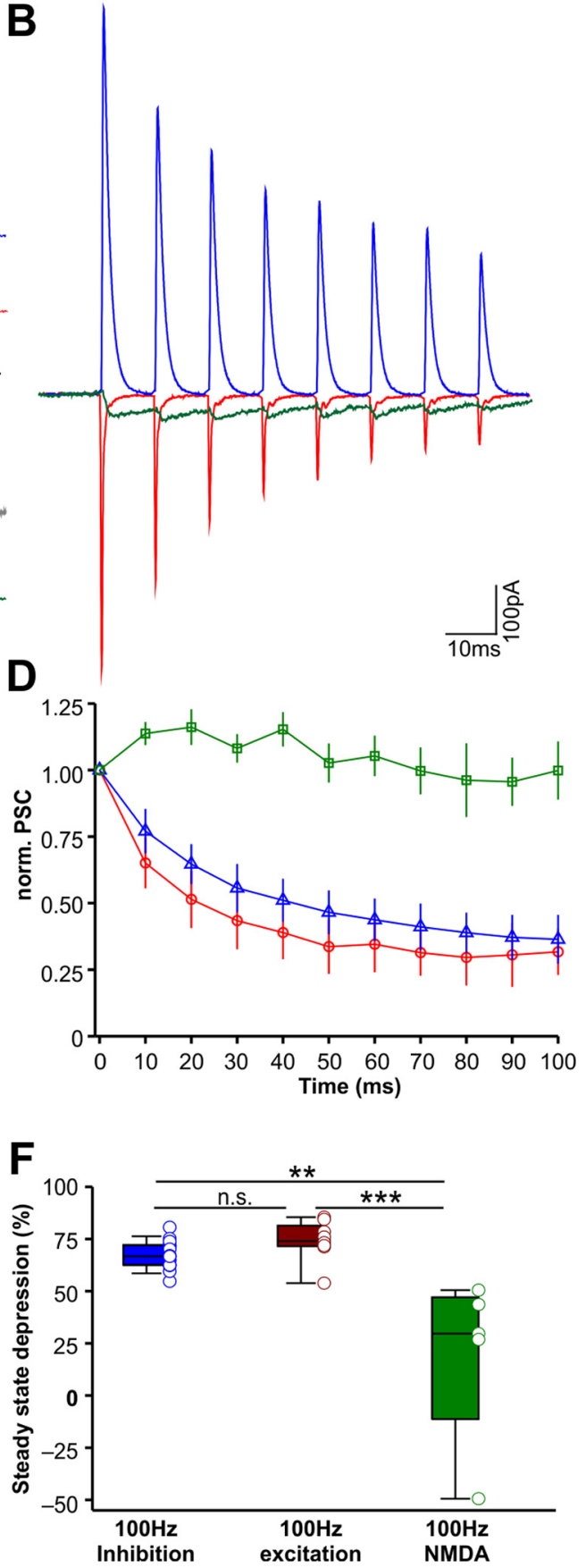

Figure 7. Short-term plasticity of excitatory and inhibitory inputs to SPN neurons. A, Examples of inhibitory (blue), excitatory (red), and NMDAR (green)-mediated responses to 50 stimulations at $100 \mathrm{~Hz}$ (averages of 10 repetitions). Gray trace depicts the lacking NMDAR mediated response at $-60 \mathrm{mV}$. B, Overlaid and magnified first responses of the examples shown in $\boldsymbol{A}$. The NMDA trace (green) has been flipped to symbolize its excitatory nature. C, Normalized and averaged current amplitudes to each of the 50 pulses of the $100-\mathrm{Hz}$ train for NMDA currents (green), EPSCs (red), and IPSCs (blue). $\boldsymbol{D}$, First $100 \mathrm{~ms}$ of the plots shown in $\boldsymbol{C}$. $\boldsymbol{E}$, Time constant $(\tau)$ of the rate of depression acquired from fitting exponential decay functions to the functions shown in $\boldsymbol{C}$. $\boldsymbol{F}$, Summary of synaptic currents steady-state depression: $100 \%$ - (average current amplitudes in response to the last five pulses $* 100$ ). $* p \leq 0.05, * * p \leq 0.01, * * * p \leq 0.001$, n.s. $=$ non-significant.

model, now including inhibition and excitation, caused neurons to fire spikes not only at the end of the stimulus train but also at the onset of stimulation (Fig. 8B). Comparing the offset responses elicited by either model revealed that the model incorporating excitation (Fig. 8C, full model) generated shorter offset-response latencies.
Adding stochastic noise to either model introduced a jitter to the offset-response latencies (Fig. 8D). However, the average latencies for the full model $(7.55 \pm 0.36 \mathrm{~ms})$ were still shorter compared to the inhibition only model (9.12 \pm $0.33 \mathrm{~ms}$ ) by $1.57 \mathrm{~ms}$ (two-tailed $t$ test: $p=0.0012 ; t=$ 3.245; DF: 998; Fig. $8 D$, red and blue vertical lines). 
A

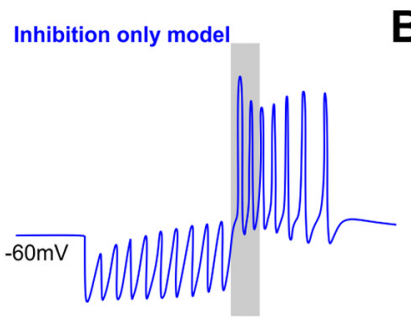

B

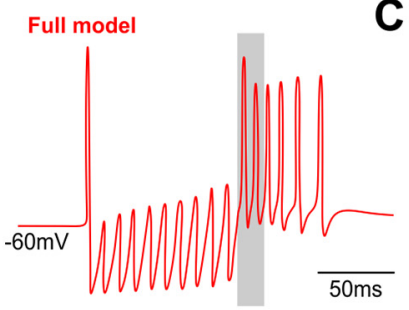

C Inhibition only model Full model

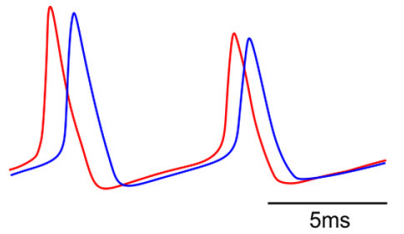

D Inhibition only model

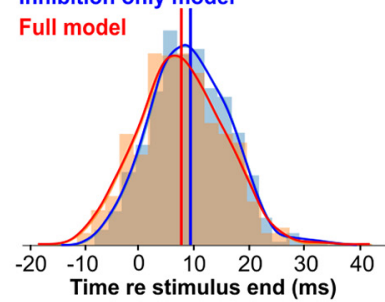

E

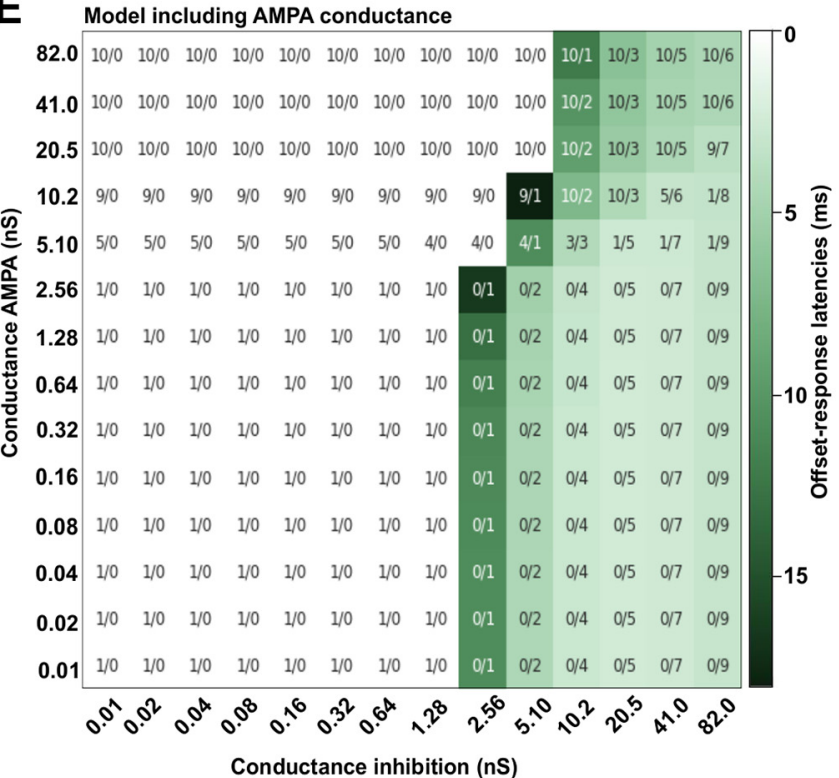

F Model including AMPA \& NMDA conductances

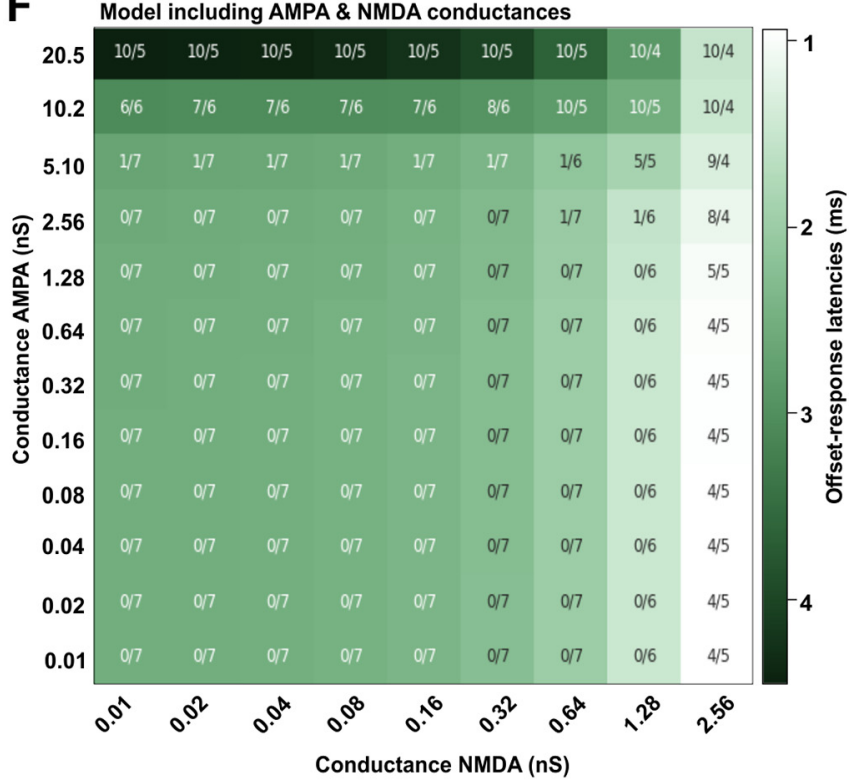

Figure 8. Excitatory input modulates offset-response timing in a computational model of SPN neurons. A, Voltage trace of a SPN neuron's response to a train of 10 stimuli using an inhibition only model shows 10 IPSPs followed by a burst of spikes at stimulus offset. $\boldsymbol{B}$, Voltage trace of a SPN neuron's response to a train of 10 stimuli using the amended "full" model including AMPAR-mediated and NMDAR-mediated excitation shows a spike at stimulus onset followed by 10 IPSPs and then by a burst of spikes at stimulus offset. $\boldsymbol{C}$, Larger temporal resolution of the first two action potentials of the offset response (gray shaded area in $\boldsymbol{A}, \boldsymbol{B}$ ). $\boldsymbol{D}$, Distributions of offset-response latencies for the inhibition only model (blue) and the full model (red) for 500 repetitions when stochastic noise was added. The red and blue solid lines represent the mean offset latencies for the full and inhibition only model, respectively. $\boldsymbol{E}$, Heat map showing changes in offset-response latencies in relation to the strength of inhibition ( $x$-axis) and AMPAR-mediated excitation ( $y$-axis). The first number for each stimulus combination depicts the number of peristimulus spikes followed by the number of poststimulus spikes in the offset response. Darker green stands for longer latencies and lighter green for shorter latencies. $\boldsymbol{F}$, Heat map showing changes in offset-response latencies in relation to the strength of AMPAR-mediated responses ( $y$-axis) and NMDAR-mediated responses ( $x$-axis). Numbers and color code are the same as in $\boldsymbol{E}$. White areas in $\boldsymbol{E}$ specify stimulus combinations that do not generate offset responses.

To assess how the balance between excitation and inhibition affects offset-response latencies, both conductances were independently varied in strength and the corresponding changes in latencies shown in a heat map (Fig. 8E; model including AMPA currents). Specific combinations elicited both onset and offset responses and were thus most comparable to the ON-OFF type neuronal responses in vivo, while in most cases no onset response was generated. Comparing the offset-response latencies for vertical columns of a specific inhibitory conductance (e.g., $41 \mathrm{nS}$ in Fig. 8E) and increasing AMPA conductances revealed that varying the AMPA conductance alone mediates the spike at stimulus onset, but did not significantly shorten the latencies of spikes in the offsetresponse (Fig. 8E). On the contrary, increasing the AMPA response beyond the physiologic estimates will prolong the offset-response latencies due to the occurrence of peristimulus spikes that interfere with the refractory pe- riod of the offset-response spikes. The shortest offsetresponse latencies were obtained with a combination of strong inhibition (41 nS) coupled with both AMPA and NMDA conductances (Fig. 8F). In the latter condition, varying the AMPA conductance between 0 and $2.56 \mathrm{nS}$ $(y$-axis) did not change the offset-response latency. However, increasing the NMDA conductance ( $x$-axis) for any of these AMPA conductances increasingly shortened the offset-response latency. Increasing the AMPA conductance further ( $\geq 5.1 \mathrm{nS}$ ), caused continuous peristimulus spike firing which then prolonged offset-response latencies due to refractory interactions. A combination of inhibition and NMDA conductance alone did not have an effect on offset-response latencies (data not shown).

These simulations suggest that although offset responses can be generated by exclusively activating the chloride conductance, the slow NMDA conductance that accompanies fast AMPA-mediated responses serve to 
A

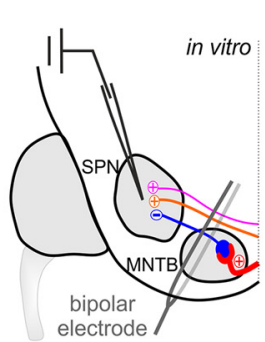

B control DNQX, AP5

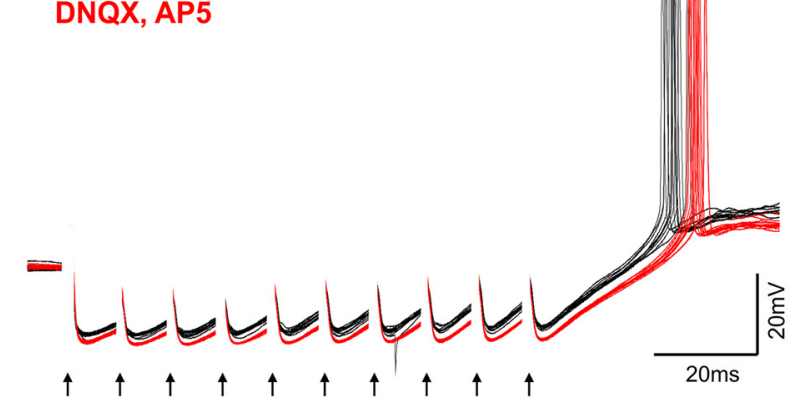

C

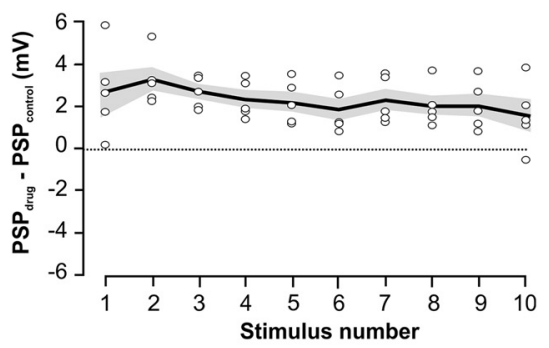

D control

E DNQX, AP5
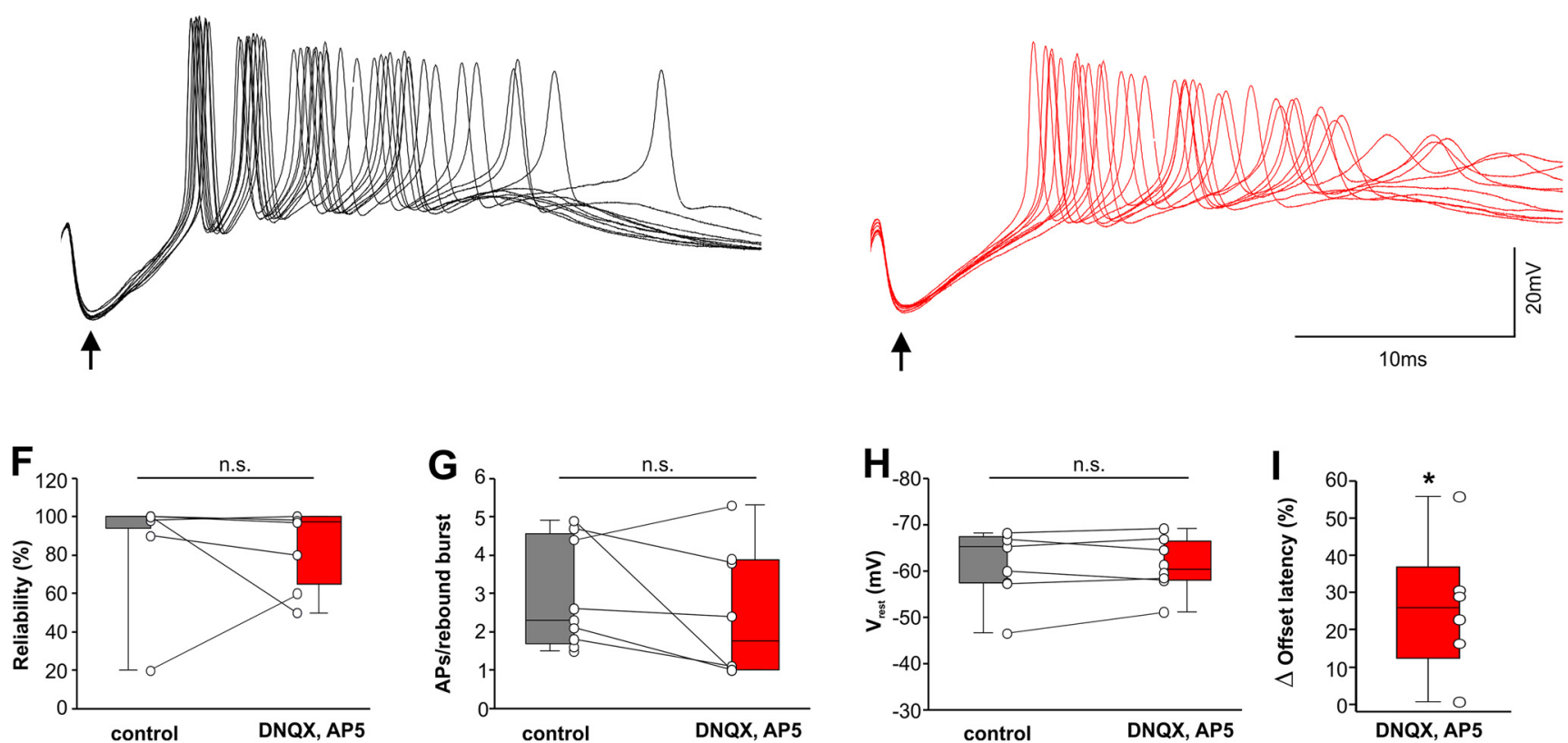

Figure 9. Excitation improves offset-response timing. $\boldsymbol{A}$, Schematic sound-offset circuit depicting the position of the fork-electrode for simultaneously stimulating excitation and inhibition. $\boldsymbol{B}, 100-\mathrm{Hz}$ synaptic stimulation (black arrows) elicited an offset response in control (black) and during blockade of excitation (red). Stimulus artifacts are removed for clarity. Postsynaptic potentials were more depolarized in control (black). C, Average difference in amplitudes of PSPs (not spikes) between blockade of excitation and control was plotted against stimulus number within each train (circles are averages of 10 trials/cell; $n=5$ cells). Black line and gray-shaded area represent mean \pm SEM. $\boldsymbol{D}, \boldsymbol{E}$, Higher temporal resolution of the offset responses of the cell shown before $(\boldsymbol{D})$ and after $(\boldsymbol{E})$ the blockade of excitation. $\boldsymbol{F}$, Reliability of offset responses in 10 consecutive traces (as shown in $\boldsymbol{D}, \boldsymbol{E} ; 100 \%$ : at least one rebound spike/trace); $n=9$. $\boldsymbol{G}$, Average number of rebound spikes (10 trials; $n=9$ ). $\boldsymbol{H}$, Resting membrane potential averaged over 10 ms before the start of synaptic stimulations $(n=9)$. I, Normalized increase in offset-response latencies after blocking excitation $(n=6) . * p \leq 0.05$, n.s. $=$ non-significant.

accelerate the offset response. Overall, a longer EPSC decay time results in a shorter latency, as the effect of the excitation is carried forward into the rebound, providing additional excitatory drive. We found that the shortest offset-response latency was generated with simulated EPSC decay time constants of $30 \mathrm{~ms}$, but even EPSC decay times of $10 \mathrm{~ms}$ accelerate the offset response.

\section{Simultaneous activation of excitation and inhibition in vitro results in a reduced net hyperpolarization of PSPs and shorter offset-response latencies}

Excitatory inputs to SPN neurons are not a prerequisite for generating offset responses (Kopp-Scheinpflug et al., 2011). However, computational modeling (Fig. 8) sug- gests that excitation might shape the temporal precision of the offset responses. Here we compared synaptically evoked offset responses in SPN neurons in control condition and during blockade of excitatory inputs by simultaneously stimulating both the MNTB and IAS using a fork-like bipolar stimulating electrode (Fig. 9A). At the end of a $100 \mathrm{~ms}, 100-\mathrm{Hz}$ train of stimuli, SPN neurons reliably generated an offset response consisting of a burst of spikes (Fig. 9B). The blockade of glutamatergic transmission during $100-\mathrm{Hz}$ stimulation for $100 \mathrm{~ms}$ (Fig. 9B, red trace) caused a hyperpolarizing drop in the net amplitude of the evoked PSPs (Fig. 9B). In control condition, the stimulation of synaptic input triggered a combined response of excitation and inhibition resulting in a net hy- 
perpolarization of $-80.9 \pm 0.3 \mathrm{mV}(n=5)$ averaged over the stimulus train. However, blocking excitation caused the PSPs to drop by $2.6 \pm 0.1 \mathrm{mV}(n=5)$ toward more hyperpolarizing voltages (Fig. 9B,C). The voltage difference between drug and control condition revealed a small, yet sustained depolarizing drive over the whole stimulus time (Fig. 9C), consistent with our simulations which predicted the presence of a small, slow excitatory conductance which extends into the temporal window of the offset response.

Blockade of excitatory inputs (only the last IPSPs of the trains shown in Fig. $9 D, E$ ) did not significantly change the reliability of generating an offset response: in 10 consecutive input trains, offset bursts were generated in $90 \pm$ $9 \%$ of trains in controls and $86 \pm 7 \%$ of trains during blockade of excitation (Mann-Whitney rank-sum test: $p=$ 0.551 ; Fig. 9F). The number of spikes within each burst of the offset response was also not significantly different between the control condition $(2.30 ; 1.7 / 4.6 ; n=9)$ and following the blockade of excitation $(1.75 ; 1.0 / 3.8 ; n=8$; Mann-Whitney rank-sum test: $p=0.359$; Fig. 9G). To probe whether tonically active excitation is present in the SPN, we compared the resting membrane potential in absence of synaptic stimulation before and during blockade of excitation, but no significant difference was found $\left(\mathrm{V}_{\text {rest }}\right.$ control: $-61.8 \pm 2.4 \mathrm{mV} ; \mathrm{V}_{\text {rest }} \mathrm{drug}:-61.1 \pm 2.0 \mathrm{mV}$; two-tailed $t$ test: $p=0.832 ; t=0.215$; df $=15$; Fig. $9 H$ ). Functionally, the blockade of excitation caused longer offset-response latencies. Depending on the exact positioning of the stimulating electrode and patch pipette in each in vitro preparation, the latencies of synapticallyevoked offset-responses varied between cells from 5.6 to $68.5 \mathrm{~ms}$ (control) and from 7.2 to $84.2 \mathrm{~ms}$ (DNQX/AP5). However, each paired recording showed an increase in latency during blockade of excitation and resulted in a significant increase of $30.68 \pm 6.71 \%$ in offset-response latency when excitation was blocked (two-tailed paired $t$ test: $p=0.01 ; t=-4.571$; df $=4$; Fig. 9/).

In conclusion, our data show that the right balance of moderate, slow excitation and strong inhibition will accelerate acoustically-evoked offset responses. Besides this faster offset firing, the presence of additional excitation also significantly reduces intensity-dependent changes in response latency. Based on the distributions of synaptic inputs within the SPN, similar CFs, thresholds and spontaneous rates between neurons with ON-OFF type and OFF-only type responses, we conclude that the different response patterns are not arising from two different types of neurons but rather reflect differences in the balance of excitation and inhibition.

\section{Discussion}

SPN neurons are reliable detectors of sound offsets and respond with a burst of spikes time-locked to the end of the stimulus. The latencies of these offset responses are short and level-invariant over a large range of suprathreshold sound intensities, which are prerequisites for sound-duration encoding and gap-detection (Forrest and Green, 1987; Brand et al., 2000; Faure et al., 2003; PérezGonzález et al., 2006). For half of the neurons with offset responses, additional excitatory inputs were observed in in vivo recordings. These neurons exhibited even shorter offset-response latencies and stronger level invariance of less than $10 \mu \mathrm{s} / \mathrm{dB}$. Pharmacological manipulation and computational modeling showed that while inhibition alone can reliably trigger a post-inhibitory rebound response at stimulus offset; excitation alone will not generate an offset response. However, the presence of slow, NMDAR-mediated excitation facilitates the rebound depolarization and speeds up the latencies of offset responses.

\section{Why don't all SPN offset cells show peristimulus excitation?}

Shorter latencies and greater resistance to level dependent latency shifts due to additional excitation provide advantages for computation of sound duration and for detection of silent gaps in noise. So why do not all SPN neurons with offset responses benefit from this advantage? Our immunocytochemical data (Fig. 1) show uniform distributions of excitatory and inhibitory inputs throughout the SPN, suggesting that the observed differences in the strength of excitation and inhibition between ON-OFF type and OFF-only type neurons may not manifest in different synaptic input patterns. This is in agreement with the lack of differences in CFs, spontaneous rates or thresholds between ON-OFF type and OFF-only type neurons. Whether or not neurons in the SPN could be classified into distinct subpopulation has been discussed for 40 years. Ollo and Schwartz (1979) used Golgi impregnations to assess the morphology of mouse SPN neurons. They described slight morphologic differences such as triangular, elongated or polygonal shapes, but stated that these were "not sufficiently distinct to warrant division into different cell types." More complex approaches combined the morphologic description with either immunostaining for the neurotransmitter used by SPN neurons (Helfert et al., 1989) or with neural tracing experiments (Schofield, 1991). As a result five SPN cell types that project to the ipsilateral inferior colliculus (IC) were described: (1) large round glycinergic neurons, (2) large round GABAergic neurons, (3) small, round, projecting bilaterally to IC glycine-negative neurons, (4) small neurons with only ipsilateral IC projections, (5) small neurons with ipsilateral IC and contralateral cochlear nucleus projections (Helfert et al., 1989; Schofield, 1991). Another approach to classify SPN neurons was taken by Felix and co-authors, who reported only subtle differences in the intrinsic properties of SPN neurons and suggested that these might be caused by gradients of potassium currents (Felix et al., 2013). Whether or not the bursting cells in the dorsolateral SPN region can form a particular subtype or are still subject to developmental change is not yet clear. In conclusion, the occurrence of ON-OFF type responses in a subset of SPN offset cells could be the result of differences in the balance of existing excitatory and inhibitory inputs. Previous studies have shown that the assessment of the excitatory-inhibitory balance might be confounded through the use of anesthetics which either block NMDA currents (ketamine-based anesthesia) or alter inhibition 
(barbiturate based anesthesia) (Steinert et al., 2008; Felix et al., 2012). Here, we used a fentanyl-based anesthesia, which binds $\mu$-opioid receptors and therefore should not directly interfere with either excitation or inhibition in the auditory brainstem.

We conclude that the occurrence of ON-OFF type and OFF-only type neurons in SPN is neither a developmental nor an experimental (anesthesia-based) effect. Instead, ON-OFF or OFF-only responses in SPN neurons are likely caused by differences in the strength of excitation and inhibition whose activity- or context-dependent control will have to be investigated to further our knowledge on encoding sound offsets.

\section{Facilitating the post-inhibitory rebound}

The excitatory input alone does not generate offset action potentials, yet it is sufficient to modify the offset responses generated via a post-inhibitory rebound mechanism (Felix et al., 2011; Kopp-Scheinpflug et al., 2011). A subthreshold inhibitory input aiding a subthreshold excitatory input to increase temporal precision is generally referred to as post-inhibitory facilitation and has been shown in other SOC neuron types (Dodla et al., 2006; Beiderbeck et al., 2018). This is not the case in our present SPN data, since here the inhibitory input on its own is sufficient to generate offset spikes. However, the slower time course of the additional excitation mediated via NMDARs allows it to extend its depolarization into the temporal window of the post-inhibitory rebound just enough to accelerate the offset response. Such an extension of excitation into the post-inhibitory rebound would require sustained excitatory responses throughout the duration of the stimulus. The fact that the peristimulus excitatory responses observed in the ON-OFF type neurons in vivo occur as either sustained $(38 \%)$ or onset $(62 \%)$ responses is likely due to most of the excitatory inputs being subthreshold caused by dominant inhibition during sound presentation, rendering subthreshold EPSPs invisible to our single cell extracellular assessment of spiking. Such subthreshold, peristimulus excitation was demonstrated by pharmacological blockade of inhibition in vivo (Kulesza et al., 2007). Based on these previous findings and our present results, it seems likely that, at least part of the peristimulus excitatory inputs are subthreshold and may serve modulatory functions rather than to form a reliable representation of sound onset. This would be in agreement with the suggestion that sound onsets and offsets are encoded in segregated pathways within the auditory brain (Scholl et al., 2010; Anderson and Linden, 2016; Kopp-Scheinpflug et al., 2018; Sollini et al., 2018). While the SPN strongly qualifies for encoding offsets, sound onset information is likely provided by different neuronal pathways such as for example the ventral nucleus of the lateral lemniscus.

The behaviorally relevant readout in the present data set is the reduction in offset-response latencies, which can decrease gap-detection thresholds (Yassin et al., 2014). The slow, lasting excitation responsible for the faster offsets, could represent the underlying cellular equivalent to adding background noise in a behavioral gap detection task, where gap detection thresholds have been significantly decreased (better), compared with a condition when no background noise was provided (Horwitz et al., 2011).

However, faster is not always better, especially when temporal precision (as measured by jitter) is equally good. An alternative interpretation is that the modulation of offset-response latencies might present a tool to accelerate or delay the time point of the offset response depending on the balance between excitation and inhibition. Such a shift in latency could present a homeostatic adaptation to a changing balance between excitation and inhibition as might occur during aging or following acoustic trauma.

\section{Reducing the level dependency of the offset response}

The offset-response latency depends on sufficient hyperpolarization and acceleration of the membrane time constant via recruiting additional ionic conductances such as $I_{H}$ (Kopp-Scheinpflug et al., 2011). Hyperpolarization of the membrane activates $\mathrm{I}_{\mathrm{H}}$, which accelerates of the membrane time constant and shortens the offsetresponse latency. If, however, $\mathrm{I}_{\mathrm{H}}$ is already maximally activated by hyperpolarization of the membrane, any further hyperpolarization might prolong the offset-response latency as it takes the membrane longer to move from $-100 \mathrm{mV}$ (SPN IPSP reversal potential) to $+40 \mathrm{mV}$ (sodium channel activation voltage) than for example from -80 to $+40 \mathrm{mV}$. This is likely what happens with the trend of increasing offset-response latencies with increasing intensity seen in Figure $3 A, B$. At higher sound intensities, multiple MNTB axons will be recruited and due to input summation, the net-depression of inhibitory inputs in SPN neurons will be reduced, resulting in strong hyperpolarization of the membrane voltage even at the end of the stimulus train. The recruitment of additional excitatory conductances as observed in the present study only at higher stimulus intensities is likely to have a similar effect as $\mathrm{I}_{\mathrm{H}}$, in shunting the inhibition, providing a depolarizing drive and shortening the offset-response latencies.

In contrast, at lower sound intensities only few MNTB axons might be recruited to inhibit SPN neurons. These inhibitory inputs will depress over time as shown for the SPN in this study or for the LSO and MSO in other studies (Couchman et al., 2010; Walcher et al., 2011; Roberts et al., 2014) and an offset response is generated at the time point when the intrinsic depolarizing drive dominates the hyperpolarization. In the experimental in vitro condition, especially for long stimulations of several hundred milliseconds, this can lead to rebound responses even before the end of the stimulus train (unpublished observations). In vivo, such rebound response before the end of a sound was never observed, suggesting that in vivo the depression of collective inhibitory inputs is low and the hyperpolarization at the stimulus end is strong.

\section{Implications of level-invariant offset responses for gap-detection and sound-duration encoding}

The threshold for detecting brief silent gaps in noise provides a valuable analytical tool to measure temporal 
resolution in auditory processing (Moore, 1997). Gaps as short as 2-3 ms can be detected by humans (Penner, 1977 ) and rodents (Ison, 1982) and it has been suggested that to detect a 3-ms gap, auditory neurons need to encode onsets and offsets of sounds with a temporal acuity of $\sim 1 \mathrm{~ms}$ (Oertel et al., 2017). Gap-in-noise stimuli have also proven very helpful in determining the ability of the auditory system to encode sound offsets as a parameter independent of sound onsets (Pratt et al., 2005).

Behaviorally, sound offsets are an important cue for sound-duration encoding. Neurons in the auditory midbrain that are sensitive to sound duration act as coincidence detectors that only fire action potentials if excitatory postsynaptic responses evoked by the onset of sound temporally coincide with excitatory postsynaptic responses evoked by the offset of sound (Casseday et al., 1994; Aubie et al., 2009, 2012, 2014; Sayegh et al., 2011; ). Duration sensitive neurons have been classified according to their ability to preferably encode sounds of different durations and are referred to as short-pass, bandpass and long-pass duration tuned neurons. According to the current models of sound-duration encoding, offset excitation is needed in the coincidence detection bandpass mechanism but not in the anti-coincidence detection short-pass mechanism (Aubie et al., 2012). This is in agreement with previous results showing that temporally precise SPN offset responses vary with stimulus duration and provide an inhibitory projection to the auditory midbrain (Kadner et al., 2006; Kopp-Scheinpflug et al., 2011) where they are suggested to generate a post-inhibitory rebound excitation (Pollak et al., 2011). Interestingly, both the detection of gaps (Moore, 1997) and the discrimination of different sound durations (Klink and Klump, 2004) are relatively independent of changes in suprathreshold sound level. Especially the duration discrimination of longer sounds ( $>50 \mathrm{~ms}$ ) has been reported to not show an intensity effect (Henry, 1948). The level-independence and short latency of SPN offset responses over an extremely large range of intensities as shown in the present study provide a perfect function for the ON-OFF type SPN neurons in the encoding of longer sound durations. Deficits in SPN offset encoding might therefore result in difficulties in processing sound offsets in downstream auditory areas like the IC or the MGB (Anderson and Linden, 2016).

\section{References}

Alamilla J, Gillespie DC (2011) Glutamatergic inputs and glutamatereleasing immature inhibitory inputs activate a shared postsynaptic receptor population in lateral superior olive. Neuroscience 196: 285-296.

Anderson LA, Linden JF (2016) Mind the gap: two dissociable mechanisms of temporal processing in the auditory system. J Neurosci 36:1977-1995.

Aubie B, Becker S, Faure PA (2009) Computational models of millisecond level duration tuning in neural circuits. J Neurosci 29: 9255-9270.

Aubie B, Sayegh R, Faure PA (2012) Duration tuning across vertebrates. J Neurosci 32:6373-6390.

Aubie B, Sayegh R, Fremouw T, Covey E, Faure PA (2014) Decoding stimulus duration from neural responses in the auditory midbrain. J Neurophysiol 112:2432-2445.
Banks MI, Smith PH (1992) Intracellular recordings from neurobiotinlabeled cells in brain slices of the rat medial nucleus of the trapezoid body. J Neurosci 12:2819-2837.

Behrend O, Brand A, Kapfer C, Grothe B (2002) Auditory response properties in the superior paraolivary nucleus of the gerbil. $J$ Neurophysiol 87:2915-2928.

Beiderbeck B, Myoga MH, Müller NIC, Callan AR, Friauf E, Grothe B, Pecka M (2018) Precisely timed inhibition facilitates action potential firing for spatial coding in the auditory brainstem. Nat Commun 9:1771.

Brand A, Urban R, Grothe B (2000) Duration tuning in the mouse auditory midbrain. J Neurophysiol 84:1790-1799.

Casseday JH, Ehrlich D, Covey E (1994) Neural tuning for sound duration: role of inhibitory mechanisms in the inferior colliculus. Science 264:847-850.

Cavaco S, Lewicki MS (2007) Statistical modeling of intrinsic structures in impacts sounds. J Acoust Soc Am 121:3558-3568.

Couchman K, Grothe B, Felmy F (2010) Medial superior olivary neurons receive surprisingly few excitatory and inhibitory inputs with balanced strength and short-term dynamics. J Neurosci 30: 17111-17121.

Couchman K, Grothe B, Felmy F (2012) Functional localization of neurotransmitter receptors and synaptic inputs to mature neurons of the medial superior olive. J Neurophysiol 107:1186-1198.

Dehmel S, Kopp-Scheinpflug C, Dörrscheidt GJ, Rübsamen R (2002) Electrophysiological characterization of the superior paraolivary nucleus in the Mongolian gerbil. Hear Res 172:18-36.

Deneux T, Kempf A, Daret A, Ponsot E, Bathellier B (2016) Temporal asymmetries in auditory coding and perception reflect multilayered nonlinearities. Nat Commun 7:12682.

Denève S, Machens CK (2016) Efficient codes and balanced networks. Nat Neurosci 19:375-382.

Dodla R, Svirskis G, Rinzel J (2006) Well-timed, brief inhibition can promote spiking: postinhibitory facilitation. J Neurophysiol 95: 2664-2677.

Eggermont JJ (2015) Auditory temporal processing and its disorders. New York: Oxford University Press.

Elgueda D, Duque D, Radtke-Schuller S, Yin P, David SV, Shamma SA, Fritz JB (2019) State-dependent encoding of sound and behavioral meaning in a tertiary region of the ferret auditory cortex. Nat Neurosci 22:447-459.

Faure PA, Fremouw T, Casseday JH, Covey E (2003) Temporal masking reveals properties of sound-evoked inhibition in durationtuned neurons of the inferior colliculus. J Neurosci 23:3052-3065.

Felix RA 2nd, Fridberger A, Leijon S, Berrebi AS, Magnusson AK (2011) Sound rhythms are encoded by postinhibitory rebound spiking in the superior paraolivary nucleus. J Neurosci 31:1256612578.

Felix RA 2nd, Kadner A, Berrebi AS (2012) Effects of ketamine on response properties of neurons in the superior paraolivary nucleus of the mouse. Neuroscience 201:307-319.

Felix RA 2nd, Vonderschen K, Berrebi AS, Magnusson AK (2013) Development of on-off spiking in superior paraolivary nucleus neurons of the mouse. J Neurophysiol 109:2691-2704.

Felix RA 2nd, Magnusson AK (2016) Development of excitatory synaptic transmission to the superior paraolivary and lateral superior olivary nuclei optimizes differential decoding strategies. Neuroscience 334:1-12.

Felix RA 2nd, Gourévitch B, Gómez-Álvarez M, Leijon SCM, Saldaña E, Magnusson AK (2017) Octopus cells in the posteroventral cochlear nucleus provide the main excitatory input to the superior paraolivary Nucleus. Front Neural Circuits 11:37.

Felix RA 2nd, Gourévitch B, Portfors CV (2018) Subcortical pathways: towards a better understanding of auditory disorders. Hear Res 362:48-60.

Forrest TG, Green DM (1987) Detection of partially filled gaps in noise and the temporal modulation transfer function. J Acoust Soc Am 82:1933-1943.

Gómez-Álvarez M, Gourévitch B, Felix RA 2nd, Nyberg T, Hernández-Montiel HL, Magnusson AK (2018) Temporal informa- 
tion in tones, broadband noise, and natural vocalizations is conveyed by differential spiking responses in the superior paraolivary nucleus. Eur J Neurosci 48:2030-2049.

Grothe B (1994) Interaction of excitation and inhibition in processing of pure tone and amplitude-modulated stimuli in the medial superior olive of the mustached bat. J Neurophysiol 71:706-721.

Guinan JJ Jr, Guinan SS, Norris BE (1972) Single auditory units in the superior olivary complex I: responses to sounds and classifications based on physiological properties. Int J Neurosci 4:101-120.

Guzman SJ, Schlögl A, Schmidt-Hieber C (2014) Stimfit: quantifying electrophysiological data with Python. Front Neuroinform 8:16.

Helfert RH, Bonneau JM, Wenthold RJ, Altschuler RA (1989) GABA and glycine immunoreactivity in the guinea pig superior olivary complex. Brain Res 501:269-286.

Henry FM (1948) Discrimination of the duration of a sound. J Exp Psychol 38:734-743.

Hines ML, Carnevale NT (2001) NEURON: a tool for neuroscientists. Neuroscientist 7:123-135.

Horwitz AR, Ahlstrom JB, Dubno JR (2011) Level-dependent changes in detection of temporal gaps in noise markers by adults with normal and impaired hearing. J Acoust Soc Am 130:29282938.

Ison JR (1982) Temporal acuity in auditory function in the rat: reflex inhibition by brief gaps in noise. J Comp Physiol Psychol 96:945954.

Jalabi W, Kopp-Scheinpflug C, Allen PD, Schiavon E, DiGiacomo RR, Forsythe ID, Maricich SM (2013) Sound localization ability and glycinergic innervation of the superior olivary complex persist after genetic deletion of the medial nucleus of the trapezoid body. $J$ Neurosci 33:15044-15049.

Kadner A, Kulesza RJ Jr, Berrebi AS (2006) Neurons in the medial nucleus of the trapezoid body and superior paraolivary nucleus of the rat may play a role in sound duration coding. $J$ Neurophysiol 95:1499-1508.

Kitzes LM, Gibson MM, Rose JE, Hind JE (1978) Initial discharge latency and threshold considerations for some neurons in cochlear nuclear complex of the cat. J Neurophysiol 41:1165-1182.

Klink KB, Klump GM (2004) Duration discrimination in the mouse (Mus musculus). J Comp Physiol A Neuroethol Sens Neural Behav Physiol 190:1039-1046.

Klug A, Khan A, Burger RM, Bauer EE, Hurley LM, Yang L, Grothe B, Halvorsen MB, Park TJ (2000) Latency as a function of intensity in auditory neurons: influences of central processing. Hear Res 148: 107-123.

Kopp-Scheinpflug C, Tolnai S, Malmierca MS, Rübsamen R (2008) The medial nucleus of the trapezoid body: comparative physiology. Neuroscience 154:160-170.

Kopp-Scheinpflug C, Tozer AJ, Robinson SW, Tempel BL, Hennig $\mathrm{MH}$, Forsythe ID (2011) The sound of silence: ionic mechanisms encoding sound termination. Neuron 71:911-925.

Kopp-Scheinpflug C, Sinclair JL, Linden JF (2018) When sound stops: offset responses in the auditory system. Trends Neurosci 41:712-728.

Kulesza RJ Jr (2008) Cytoarchitecture of the human superior olivary complex: nuclei of the trapezoid body and posterior tier. Hear Res 241:52-63.

Kulesza RJ Jr, Spirou GA, Berrebi AS (2003) Physiological response properties of neurons in the superior paraolivary nucleus of the rat. J Neurophysiol 89:2299-2312.

Kulesza RJ Jr, Kadner A, Berrebi AS (2007) Distinct roles for glycine and GABA in shaping the response properties of neurons in the superior paraolivary nucleus of the rat. J Neurophysiol 97:16101620.

Kuwada S, Batra R (1999) Coding of sound envelopes by inhibitory rebound in neurons of the superior olivary complex in the unanesthetized rabbit. J Neurosci 19:2273-2287.

Löhrke S, Srinivasan G, Oberhofer M, Doncheva E, Friauf E (2005) Shift from depolarizing to hyperpolarizing glycine action occurs at different perinatal ages in superior olivary complex nuclei. Eur $\mathrm{J}$ Neurosci 22:2708-2722.
Moore BCJ (1997) An introduction to the psychology of hearing, Ed 4. San Diego, CA: Academic Press.

Morgan MJ, Thompson P (1975) Apparent motion and the Pulfrich effect. Perception 4:3-18.

Mountcastle VB, Davies PW, Berman AL (1957) Response properties of neurons of cat's somatic sensory cortex to peripheral stimuli. J Neurophysiol 20:374-407.

Oertel D, Cao XJ, Ison JR, Allen PD (2017) Cellular computations underlying detection of gaps in sounds and lateralizing sound sources. Trends Neurosci 40:613-624.

Ollo C, Schwartz IR (1979) The superior olivary complex in C57BL/6 mice. Am J Anat 155:349-373.

Penner MJ (1977) Detection of temporal gaps in noise as a measure of the decay of auditory sensation. J Acoust Soc Am 61:552-557.

Pérez-González D, Malmierca MS, Moore JM, Hernández O, Covey $E$ (2006) Duration selective neurons in the inferior colliculus of the rat: topographic distribution and relation of duration sensitivity to other response properties. J Neurophysiol 95:823-836.

Phillips DP, Hall SE, Boehnke SE (2002) Central auditory onset responses, and temporal asymmetries in auditory perception. Hear Res 167:192-205

Pilati N, Linley DM, Selvaskandan H, Uchitel O, Hennig MH, KoppScheinpflug C, Forsythe ID (2016) Acoustic trauma slows AMPA receptor-mediated EPSCs in the auditory brainstem, reducing GluA4 subunit expression as a mechanism to rescue binaural function. J Physiol 594:3683-3703.

Pollak GD, Gittelman JX, Li N, Xie R (2011) Inhibitory projections from the ventral nucleus of the lateral lemniscus and superior paraolivary nucleus create directional selectivity of frequency modulations in the inferior colliculus: a comparison of bats with other mammals. Hear Res 273:134-144.

Pratt H, Bleich N, Mittelman N (2005) The composite N1 component to gaps in noise. Clin Neurophysiol 116:2648-2663.

Rhode WS, Oertel D, Smith PH (1983) Physiological response properties of cells labeled intracellularly with horseradish peroxidase in cat ventral cochlear nucleus. J Comp Neurol 213:448-463.

Roberts MT, Seeman SC, Golding NL (2014) The relative contributions of MNTB and LNTB neurons to inhibition in the medial superior olive assessed through single and paired recordings. Front Neural Circuits 8:49.

Saldaña E, Aparicio MA, Fuentes-Santamaría V, Berrebi AS (2009) Connections of the superior paraolivary nucleus of the rat: projections to the inferior colliculus. Neuroscience 163:372-387.

Sayegh R, Aubie B, Faure PA (2011) Duration tuning in the auditory midbrain of echolocating and non-echolocating vertebrates. $J$ Comp Physiol A Neuroethol Sens Neural Behav Physiol Neuroethol Sens Neural Behav Physiol 197:571-583.

Schofield BR (1991) Superior paraolivary nucleus in the pigmented guinea pig: separate classes of neurons project to the inferior colliculus and the cochlear nucleus. J Comp Neurol 312: 68-76.

Schofield BR (1995) Projections from the cochlear nucleus to the superior paraolivary nucleus in guinea pigs. J Comp Neurol 360: 135-149.

Scholl B, Gao X, Wehr M (2010) Nonoverlapping sets of synapses drive on responses and off responses in auditory cortex. Neuron 65:412-421.

Smith AJ, Owens S, Forsythe ID (2000) Characterisation of inhibitory and excitatory postsynaptic currents of the rat medial superior olive. J Physiol 529 [Pt 3]:681-698.

Sohoglu E, Chait M (2016) Neural dynamics of change detection in crowded acoustic scenes. Neuroimage 126:164-172.

Sollini J, Chapuis GA, Clopath C, Chadderton P (2018) ON-OFF receptive fields in auditory cortex diverge during development and contribute to directional sweep selectivity. Nat Commun 9:2084. 
Steinert JR, Kopp-Scheinpflug C, Baker C, Challiss RA, Mistry R, Haustein MD, Griffin SJ, Tong H, Graham BP, Forsythe ID (2008) Nitric oxide is a volume transmitter regulating postsynaptic excitability at a glutamatergic synapse. Neuron 60:642656.

Steinert JR, Postlethwaite M, Jordan MD, Chernova T, Robinson SW, Forsythe ID (2010) NMDAR-mediated EPSCs are maintained and accelerate in time course during maturation of mouse and rat auditory brainstem in vitro. J Physiol 588:447-463.

Sun YJ, Wu GK, Liu BH, Li P, Zhou M, Xiao Z, Tao HW, Zhang LI (2010) Fine-tuning of pre-balanced excitation and inhibition during auditory cortical development. Nature 465:927-931.
Thompson AM, Thompson GC (1991) Posteroventral cochlear nucleus projections to olivocochlear neurons. J Comp Neurol 303:267-285.

Walcher J, Hassfurth B, Grothe B, Koch U (2011) Comparative posthearing development of inhibitory inputs to the lateral superior olive in gerbils and mice. J Neurophysiol 106:1443-1453.

Wehr M, Zador AM (2003) Balanced inhibition underlies tuning and sharpens spike timing in auditory cortex. Nature 426:442-446.

Yassin L, Radtke-Schuller S, Asraf H, Grothe B, Hershfinkel M, Forsythe ID, Kopp-Scheinpflug C (2014) Nitric oxide signaling modulates synaptic inhibition in the superior paraolivary nucleus (SPN) via cGMP-dependent suppression of KCC2. Front Neural Circuits 8:65. 